\title{
An AICD-based functional screen to identify APP metabolism regulators
}

\author{
Can Zhang ${ }^{\dagger}$, Preeti J Khandelwal ${ }^{\dagger 1}$, Ranjita Chakraborty ${ }^{1}$, Trinna L Cuellar ${ }^{1}$, \\ Srikant Sarangi ${ }^{1}$, Shyam A Patel ${ }^{1}$, Christopher P Cosentino ${ }^{1}$, \\ Michael O'Connor ${ }^{1}$, Jeremy C Lee ${ }^{1}$, Rudolph E Tanzi ${ }^{2}$ and \\ Aleister J Saunders*1,3
}

Address: ${ }^{1}$ Department of Bioscience \& Biotechnology, Drexel University, Philadelphia, PA, USA, ${ }^{2}$ Genetics and Aging Research Unit, MassGeneral Institute for Neurodegenerative Diseases (MIND), Department of Neurology, Massachusetts General Hospital, Harvard Medical School, Charlestown, MA, USA and ${ }^{3}$ Department of Biochemistry \& Molecular Biology, Drexel University College of Medicine, Philadelphia, PA, USA

Email: Can Zhang - cz33@drexel.edu; Preeti J Khandelwal - pjk25@drexel.edu; Ranjita Chakraborty -rm3282@drexel.edu; Trinna L Cuellar - tcuellar@diabetes.ucsf.edu; Srikant Sarangi - smg36@drexel.edu; Shyam A Patel - sap42@drexel.edu;

Christopher P Cosentino - nimblejak@aol.com; Michael O'Connor - oconnomp@drexel.edu; Jeremy C Lee - jl46@drexel.edu; Rudolph E Tanzi - tanzi@helix.mgh.harvard.edu; Aleister J Saunders* - Aleister.Saunders@drexel.edu

* Corresponding author †Equal contributors

Published: 24 August 2007

Molecular Neurodegeneration 2007, 2:15 doi:10.1186/1750-1326-2-15
Received: 12 June 2007

Accepted: 24 August 2007

This article is available from: http://www.molecularneurodegeneration.com/content/2/I/I5

(C) 2007 Zhang et al; licensee BioMed Central Ltd.

This is an Open Access article distributed under the terms of the Creative Commons Attribution License (http://creativecommons.org/licenses/by/2.0), which permits unrestricted use, distribution, and reproduction in any medium, provided the original work is properly cited.

\begin{abstract}
Background: A central event in Alzheimer's disease (AD) is the regulated intramembraneous proteolysis of the $\beta$-amyloid precursor protein (APP), to generate the $\beta$-amyloid $(A \beta)$ peptide and the APP intracellular domain (AICD). A $\beta$ is the major component of amyloid plaques and AICD displays transcriptional activation properties. We have taken advantage of AICD transactivation properties to develop a genetic screen to identify regulators of APP metabolism. This screen relies on an APP-Gal4 fusion protein, which upon normal proteolysis, produces AICD-Gal4. Production of AICD-Gal4 induces Gal4-UAS driven luciferase expression. Therefore, when regulators of APP metabolism are modulated, luciferase expression is altered.
\end{abstract}

Results: To validate this experimental approach we modulated $\alpha-, \beta$-, and $\gamma$-secretase levels and activities. Changes in AICD-Gal4 levels as measured by Western blot analysis were strongly and significantly correlated to the observed changes in AICD-Gal4 mediated luciferase activity. To determine if a known regulator of APP trafficking/maturation and Presenilin I endoproteolysis could be detected using the AICD-Gal4 mediated luciferase assay, we knocked-down Ubiquilin I and observed decreased luciferase activity. We confirmed that Ubiquilin I modulated AICD-Gal4 levels by Western blot analysis and also observed that Ubiquilin I modulated total APP levels, the ratio of mature to immature APP, as well as PSI endoproteolysis.

Conclusion: Taken together, we have shown that this screen can identify known APP metabolism regulators that control proteolysis, intracellular trafficking, maturation and levels of APP and its proteolytic products. We demonstrate for the first time that Ubiquilin I regulates APP metabolism in the human neuroblastoma cell line, SH-SY5Y. 


\section{Background}

Alzheimer's disease (AD) is characterized by significant accumulation of cerebral amyloid plaques and intraneuronal neurofibrillary tangles. Amyloid plaques are composed mainly of the $\beta$-amyloid peptide $(A \beta) . A \beta$ is a normal product of amyloid precursor protein (APP) metabolism. Several genes have been identified encoding enzymes that directly metabolize APP to generate $A \beta$; however, it is not fully understood how APP metabolism is regulated. Here we describe and validate a novel experimental approach for identifying genes encoding regulators of APP metabolism.

$\mathrm{A} \beta$ is generated by the successive proteolytic processing of APP, a process referred to as regulated intramembrane proteolysis (RIP) [1-3]. RIP occurs when a transmembrane protein is cleaved within the transmembrane domain, releasing a cytoplasmic fragment that can activate gene expression in the nucleus [1]. RIP requires two cleavage events; the first, outside the membrane, often in response to ligand binding, can trigger the second, intramembraneous, cleavage. RIP liberates small, intracellular protein domains that are involved in nuclear signaling processes $[1,2]$. Therefore, regulation of RIP is critical for controlling nuclear signaling. Identifying the regulatory mechanisms controlling these proteolytic steps is important for a fuller understanding of these processes.

APP is a type I transmembrane glycoprotein and is suggested to function in neuroprotection, synaptic transmission, signal transduction, and axonal transport $[4,5]$. Upon being synthesized, APP undergoes maturation in the protein secretory pathway. APP is N-glycosylated in the ER and cis-Golgi followed by O-glycosylation in medial- and trans-Golgi. RIP of APP can occur via two alternative routes: amyloidogenic and non-amyloidogenic. In amyloidogenic processing, APP undergoes sequential cleavage by $\beta$-secretase (BACE) and $\gamma$-secretase to generate $A \beta[6]$. BACE cleavage occurs in the APP extracellular domain to produce a soluble extracellular fragment called sAPP $\beta$ and a membrane associated, 99residue C-terminal fragment called C99 [7] The C99 fragment is a substrate for subsequent cleavage by the $\gamma$-secretase complex $[8,9]$. The active $\gamma$-secretase complex is composed of the amino- and carboxy-terminal fragments of presenilin1 (PS1), a highly glycosylated form of nicastrin (NCSTN), Aph $1 \alpha$ and Pen-2 [8,9]. The amino- and carboxy-terminal fragments of PS1 ( 27 and 17 kDa respectively) are derived by endoproteolytic cleavage of the inactive, full length PS1 protein within the large hydrophilic loop that spans between transmembrane helices 6 and 7 and are thought to interact with each other [10]. The products of $\gamma$-secretase cleavage are the cytoplasmic APP Intracellular Domain (AICD) fragment and A $\beta$ peptides of varying length, mainly 40 and 42 residues long [11-13]. In non-amyloidogenic processing, the initial extracellular cleavage of APP is catalyzed by one of a group of proteases termed $\alpha$-secretases. These enzymes include ADAM9, ADAM10, and ADAM17 (TACE). $\alpha$ secretase cleavage produces a soluble extracellular fragment called sAPP $\alpha$ and a membrane associated, 83-residue C-terminal fragment called C83. This C83 fragment is then cleaved by the $\gamma$-secretase complex to produce AICD and a p3 peptide, which is not involved in amyloidogenesis [6].

A common feature of RIP processing is the liberation of an intracellular protein domain that initiates nuclear signaling $[1,2]$. In the case of APP processing, nuclear signaling can be initiated by the production of the intracellular AICD fragment. Once generated by $\gamma$-secretase, the AICD fragment can be stabilized and transported to the nucleus by the cytoplasmic adaptor protein Fe65 [14,15]. Upon entering the nucleus the AICD/Fe65 complex can form a tripartite, transcriptionally active complex with the histone acetyltransferase Tip60 [16,17]. Consistent with this model, cells concomitantly over-expressing an APP-Gal4DNA binding domain fusion protein and Fe65, and carrying a Gal4 UAS-driven reporter construct display a >2000 fold increase in reporter transcription compared to cells over-expressing just the Gal4 DNA binding domain and Fe65 [16]. This increase in transactivation activity is dependent on Tip60 and can be abolished when the interaction between AICD and Fe65 is disrupted by mutagenesis of the AICD NPTY motif, the binding site for Fe65 [16]. However, these data do not rule out a possible effect of full-length APP in inducing nuclear signaling. Indeed, APP nuclear signaling can occur in the absence of $\gamma$-secretase activity and therefore does not require the AICD fragment [18]. The relative contribution of AICD-mediated versus holo-APP mediated nuclear signaling is not clear at this time [16-18].

The genomic targets of AICD- or APP-mediated nuclear signaling are not clearly defined. APP, BACE, Tip60, GSK$3 \beta, M n-S O D, K A I 1, N E P$ and other genes have all been reported to be targets of APP mediated transcriptional activation [19-22]; however, there is a paucity of confirmatory reports [18]. At this time, the biological role of AICD-mediated transactivation is not clear $[20,23,24]$. Despite this confusion, evidence suggests that defective APP signaling is involved in AD pathogenesis [25-29].

Given the centrality of APP in AD, it is crucial to identify regulators of APP metabolism, including, but not limited to, APP proteolysis. Regulation of APP metabolism can occur by numerous mechanisms, including regulation of APP transcription, APP translation, APP maturation, intracellular trafficking of full-length APP and APP cleavage products, APP proteolysis, and APP degradation. While 
Komano and colleagues have used a genetic screen to specifically identify regulators of $\gamma$-secretase activity [30], a screen that will identify APP metabolism regulators that act through multiple mechanisms is needed.

Here we describe a novel experimental approach to identify a variety of regulators of APP metabolism. We use an AICD-Gal4 mediated luciferase expression assay as a general reporter of APP metabolism in the human neuroblastoma cell lines, SH-SY5Y. To validate this assay, we utilized pharmacologic agents, as well as forward and reverse genetics, to modulate APP proteolysis, AICD trafficking and AICD transactivation. To determine if regulators of APP maturation and PS1 endoproteolysis also can be detected with this screening approach, we knockeddown Ubiquilin 1 and observed decreased AICD-Gal4 luciferase activity. Using Western blot analysis, we show that Ubiquilin 1 controls APP levels, the ratio of mature to immature APP, as well as presenilin1 endoproteolysis, confirming the previously reported role of Ubiquilin 1 in APP and presenilin 1 metabolism in non-neuronal human cell lines [31-34]. Taken together, our results validate the use of the AICD-Gal4 mediated luciferase assay in combination with forward and reverse genetics as a screen to identify APP metabolism regulators.

\section{Results \\ Establishment of a functional assay to identify APP metabolism regulators}

We utilized the APP-Gal4/Gal4-UAS luciferase reporter system (Figure 1A) developed by Cao and Südhof [16]. We established this assay system in our laboratory by creating a SH-SY5Y, human neuroblastoma, cell line that stably expresses the assay components. Three different stable cell lines have been generated; all stably carry a luciferase reporter gene under the control of the Gal4-UAS (Gal4UAS luciferase). In addition to this reporter gene, one cell line expresses the Gal4 DNA binding domain alone (SY5Y-Gal4), the second cell line expresses $\mathrm{APP}_{695}$ fused to the Gal4 DNA binding domain (SY5Y-APP-Gal4), and the third cell line expresses a mutated version of $\mathrm{APP}_{695^{-}}$ Gal4 (SY5Y-APP*-Gal4). This mutation in APP alters the NPTY motif (P685A; Y687A) and disrupts Fe65 binding to this site [16]. Once these cells were established, luciferase assays were performed to determine the relative luciferase activity of the cell lines (Figure 1B). SY5Y-APP-Gal4 cells have a statistically significant $(\mathrm{p}<0.01) \sim 20$ fold increase in luciferase activity compared to SY5Y cells expressing either Gal4 or APP*-Gal4.

\section{Pharmacologic modulation of $\alpha$ - and $\gamma$-secretase activity alters AICD-Gal4 mediated luciferase activity}

To determine if monitoring AICD-Gal4 mediated luciferase activity is a valid method to detect alterations in APP metabolism, we used pharmacologic agents known to modulate APP proteolytic processing and compared the effects of these agents on levels of APP proteolytic products and AICD-Gal4 mediated luciferase activity. To accomplish this, we treated our SY5Y-APP-Gal4 cells with pharmacologic modulators of $\alpha_{-}, \beta-$, and $\gamma$-secretase activity and measured the effects using Western blot analyses for APP cleavage products as well as AICD-Gal4 mediated luciferase activity.

L-685,458 is a transition state inhibitor of $\gamma$-secretase that prevents $A \beta$ and AICD generation [35]. We treated SY5YAPP-Gal4 cells with L-685,458 (2.5 $\mu \mathrm{M}$ for 10 hours $)$ or with vehicle (DMSO) and collected cell lysates. We performed Western blot analysis on these cell lysates using an antibody to the C-terminus of human APP. In vehicle treated cells we observe bands migrating at 28 and 26 kDa (Figure 2A). Cao and Sudhof observed a similar doublet at approximately the same relative molecular weight. They identified these bands as C83-Gal4 and AICD-Gal4, respectively. In L-685,458 treated cells the intensity of the C83-Gal4 band is significantly increased seven-fold ( $\mathrm{p}<$ 0.01 ) and the AICD-Gal4 band is significantly decreased by $80 \%$ ( $p<0.01$; Figure $2 \mathrm{~B})$. These results are consistent with the substrate/product relationship between C83Gal4 and AICD-Gal4. The size difference between C83Gal4 and AICD-Gal4 is what is expected for $\gamma$-secretase cleavage of C83-Gal4. It is also interesting to note that AICD is normally difficult to detect by Western blot analysis, however the AICD-Gal4 fusion levels are quite high. This suggests that AICD-Gal4 catabolism by IDE and/or other proteases is greatly reduced compared to unmodified AICD [36].

L-685,458 treatment of SY5Y-APP-Gal4 cells results in a concentration-dependent decrease in AICD-Gal4 mediated luciferase activity; at a concentration of $2.5 \mu \mathrm{M}$ there is a $\sim 75 \%$ decrease in luciferase activity (Figure $2 \mathrm{C}$ ). The L- 685,458 concentration required for $50 \%$ inhibition of AICD-Gal4 mediated luciferase activity is $1.25 \mu \mathrm{M}$.

The phorbol ester, PMA (phorbol 12-myristate 13-acetate), stimulates $\alpha$-secretase activity [37]. Treatment of SY5Y-APP-Gal4 cells with PMA ( $1 \mu \mathrm{M}$ for 10 hours) resulted in approximately two-fold increase in the levels of the $\alpha$-secretase cleavage products sAPP $\alpha$ and C83-Gal4 $(\mathrm{p}<0.01$; Figures $3 \mathrm{~A} \& 3 \mathrm{~B})$. In addition, Western blot analysis revealed a two-fold increase in AICD-Gal4 levels $(\mathrm{p}<$ 0.01 ; Figures $3 \mathrm{~A}$ \&3B). This two-fold increase in AICDGal4 levels suggested that a similar PMA-induced increase in AICD-Gal4 mediated luciferase activity should be observed. Indeed, when we measured luciferase activity as a function of increasing PMA concentration (Figure 3C), we observed a dose-dependent increase in luciferase activity. The PMA-induced increases in luciferase activity plateaus at $50 \mathrm{nM}$ PMA. At concentrations of $50 \mathrm{nM}$ and 
A

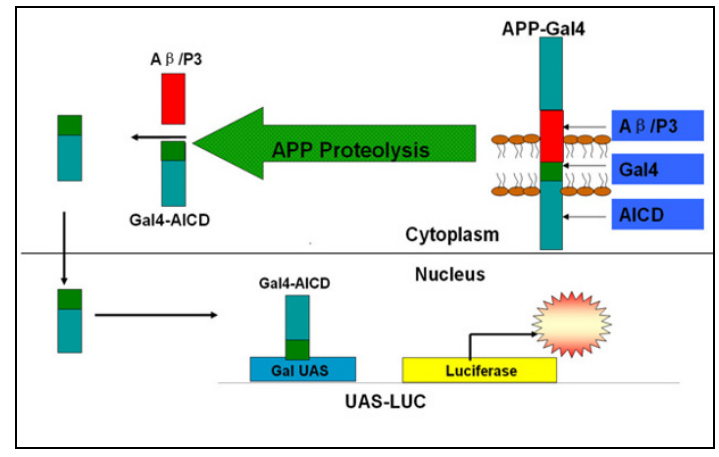

B

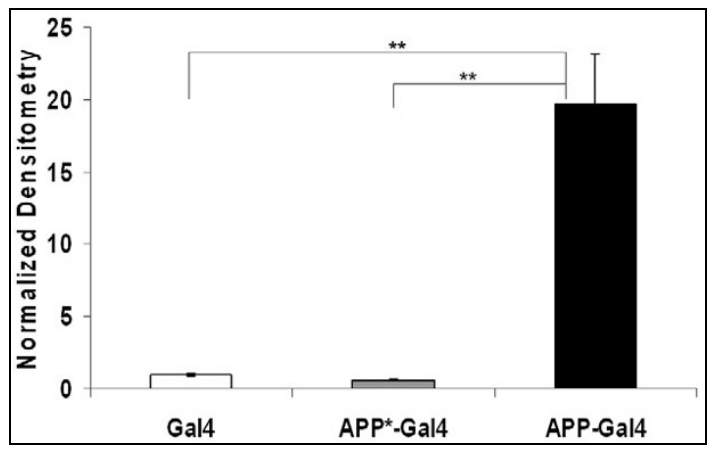

\section{Figure I}

Functional screen for regulators of APP metabolism. (A) Model depicting APP-Gal4 reporter system. (B) Firefly luciferase activity is significantly increased in SH-SY5Y cells stably expressing APP-Gal4 and Gal4-UAS Luciferase compared to SY5Y cells stably expressing either Gal4/Gal4-UAS Luciferase or APP*-Gal4/Gal4-UAS Luciferase. Luciferase activity was normalized to total cell number using SYBR Green. Bars represent mean normalized luciferase expression (+/- std. error) of 16 independent trials for each cell line. Student's t-tests with sequential Bonferroni correction for multiple comparisons; ** indicates $\mathrm{p}<0.01$.

higher, we observed approximately a two-fold increase in AICD-Gal4 mediated luciferase activity in close agreement with the observed two-fold increase in AICD-Gal4 by Western blot analysis.

TAPI-1 (Tumor necrosis factor- $\alpha$ protease inhibitor 1) inhibits $\alpha$-secretase mediated shedding of the APP ectodomain [38]. Treating the SY5Y-APP-Gal4 cells with TAPI-1 (20 $\mu \mathrm{M}$ for two hours; Figure 3D \&3E) resulted in a modest, yet significant decrease of sAPP $\alpha(31 \%, \mathrm{p}<0.05)$, C83-Gal4 (27\%, p < 0.01) and AICD-Gal4 levels (36\%; p $<0.01)$, as well as in AICD-Gal4 mediated luciferase activity $(38 \% ; \mathrm{p}<0.01)$. In addition, TAPI-1 exhibits a dosedependent effect with $20 \mu \mathrm{M}$ resulting in a $37 \%$ decrease in AICD-Gal4 mediated luciferase activity (Figure 3F). Again, these data show that alterations in AICD-Gal4 levels as detected by Western blot can be accurately detected by the AICD-Gal4 mediated luciferase assay.

Finally, we treated SY5Y-APP-Gal4 cells with a $\beta$-secretase inhibitor ( $\beta$-secretase inhibitor II). This inhibitor prevents BACE-mediated cleavage of APP and generation of A $\beta$ [39]. Treating these cells resulted in no observable change in AICD-mediated luciferase activity. This result is not surprising given the very low levels of $\beta$-secretase cleaved APP (C99-Gal4) that we observe in these cells compared to the high levels of $\alpha$-secretase cleaved APP (C83-Gal4) we observe (Figures 2A, 3A, 3D). We estimate that of all the APP molecules undergoing $\alpha$ - or $\beta$-secretase cleavage only about $10 \%$ are cleaved by $\beta$-secretase, using our Western blot data (data not shown). Therefore, inhibition of BACE, even if effective, may result in an undetectable change in the levels of cleavage products in this experimental scheme.

In summary, pharmacologic modulation of $\alpha$ - and $\gamma$ secretase activities alters AICD-Gal4 mediated luciferase activities that accurately correspond to the changes in AICD-Gal4 levels determined by Western blot analysis.

\section{Genetic manipulation of secretase levels modulates AICD- Gal4 mediated luciferase activity}

To further validate the AICD-Gal4 mediated luciferase assay as a reporter of APP metabolism, we over-expressed and knocked-down the expression of genes involved in $\alpha$ , $\beta$ - \& $\gamma$-secretase activities. Again, we compared the effects of over-expression or knock-down on levels of APP proteolytic products quantified by Western blot analysis to AICD-Gal4 mediated luciferase activity in SY5Y-APP-Gal4 cells.

Over-expression experiments were conducted by transiently transfecting individual over-expression plasmids or empty vector controls into the SY5Y-APP-Gal4 cells. Cell lysates and conditioned media were collected $24-48$ hours post transfection. ADAM10 and ADAM17 overexpression promoted $\alpha$-secretase cleavage of APP and increased sAPP $\alpha$ secretion as compared to "empty vector" transfected cells (Figure 4A - 4D). ADAM10 and ADAM17 over-expression also significantly increased C83-Gal4 and 
A

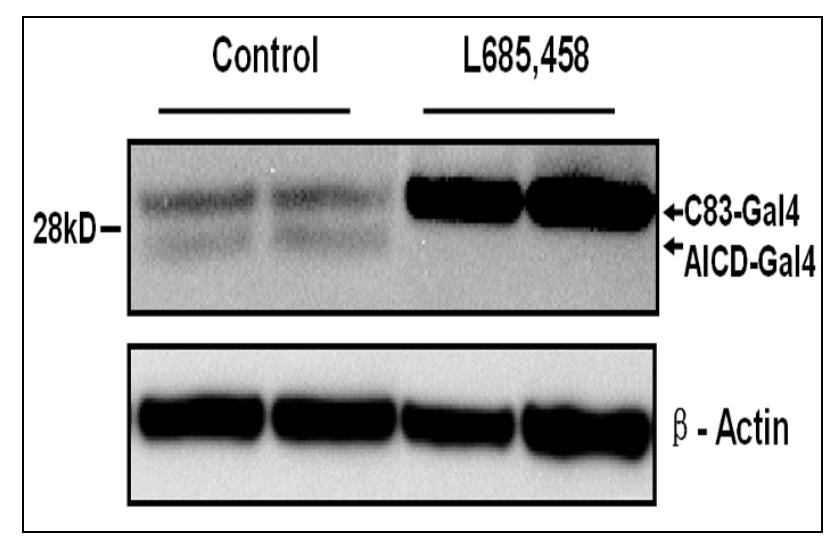

C
B

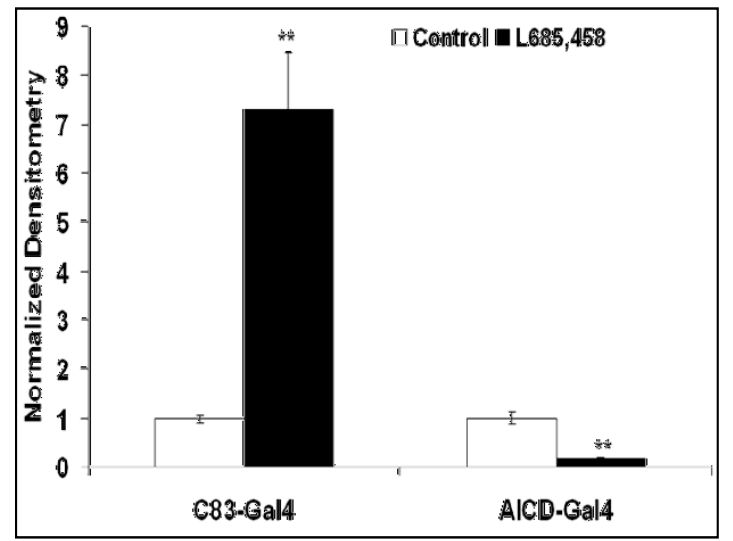

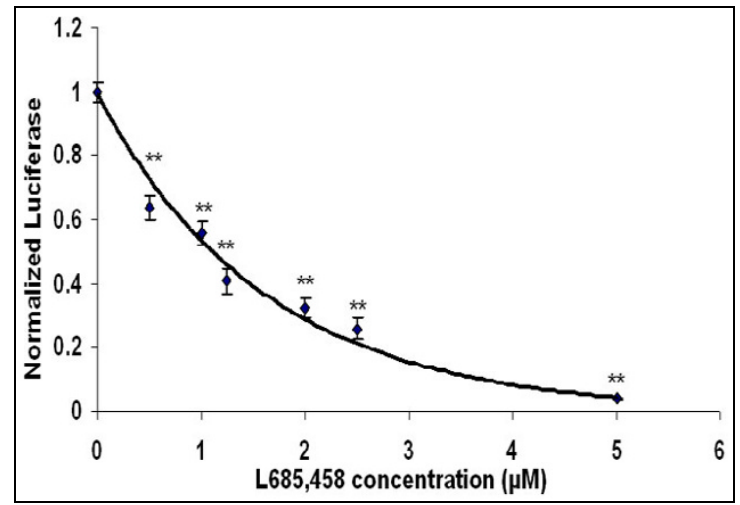

Figure 2

$\gamma$-secretase inhibition decreases AICD-Gal4 levels and AICD-Gal4 mediated luciferase activity in SY5Y-APPGal4 cells. (A) Inhibition of $\gamma$-secretase by L-685,458 (5 mM) decreases AICD-Gal4 levels and increases C83-Gal4 levels as detected by Western blot analysis. (B) Quantification of Western blot densitometry in panel A. Normalization for loading differences was achieved by dividing the densitometry values for individual bands by the densitometry values for $\beta$-actin in the same lane. (C) Dose-dependent decreases in AICD-Gal4-mediated luciferase activity with increasing concentrations of L685,458 . For the luciferase experiments, points represent mean normalized luciferase activity $(+/$ - standard error) of three independent trials, with luciferase levels normalized to total cell numbers using SYBR Green. Student's t-tests with sequential Bonferroni correction for multiple comparisons was utilized to test for significance. * indicates $p<0.05$; ** indicates $p<0.0$ I. "Control" uses the same media as the treatments, and also contains the same amount of DMSO.

AICD-Gal4 levels as detected by Western blot (Figure 4A 4D). Specifically, AICD-Gal4 levels increased approximately three-fold for both (Figure 4B \&4D). Measuring AICD-Gal4 mediated luciferase activity, we found that over-expression of ADAM10 and ADAM17 resulted in a statistically significant three to five fold increase in luciferase activity (Figure 4E). Furthermore, over-expression of the $\beta$-secretase gene (BACE) or individual components of the $\gamma$-secretase complex (PSEN1, PEN2, APH1, and NCSTN) or another $\alpha$-secretase member ADAM9 also results in increased luciferase activity (Figure 4E). Specifically, BACE over-expression significantly increased luci- ferase activity approximately two fold $(\mathrm{p}<0.01)$, PEN2 and NCSTN over-expression increased luciferase activity up to two fold $(\mathrm{p}<0.01$.) However, over-expression of PSEN1 and APH1 did not result in any significant change in luciferase activity.

We knocked-down the genes responsible for $\alpha$ - and $\gamma$ secretase using commercially available shRNAs [40]. A control shRNA, which is not complementary to any known human gene, was used as a negative control. SY5YAPP-Gal4 cells were transfected with individual shRNAs and selected with $2 \mu \mathrm{g} / \mathrm{ml}$ puromycin for 5 to 7 days. Con- 
A

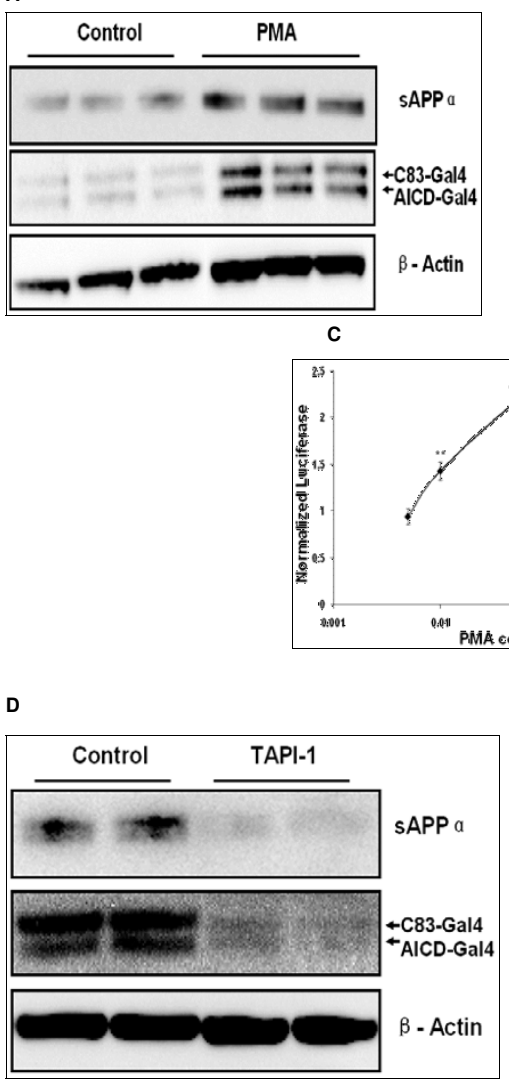

B

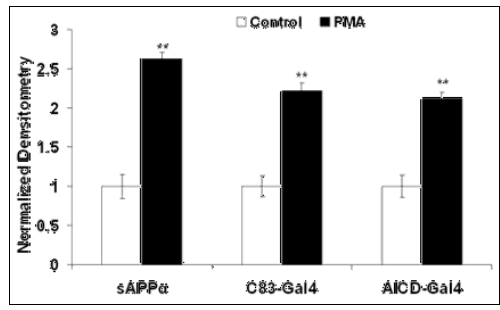

F

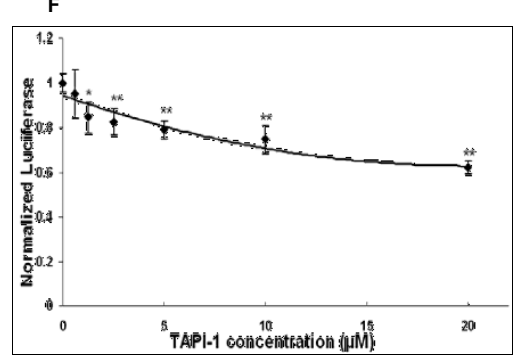

E

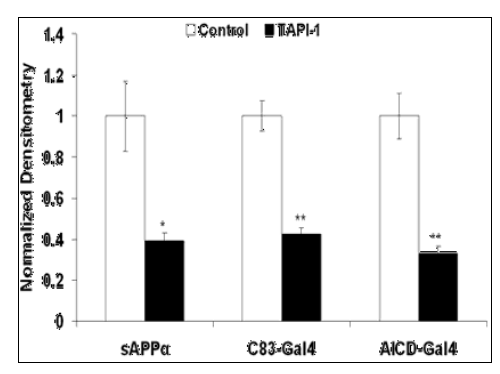

concentitation:

Figure 3

Pharmacological modulation of $\alpha$-secretase activity alters AICD-Gal4 levels and AICD-Gal4 mediated luciferase activity in SY5Y-APP-Gal4 cells. (A) Stimulation of $\alpha$-secretase by PMA (I $\mu$ M PMA for I0 hours) increases sAPP $\alpha$, C83-Gal4, and AICD-Gal4 levels as detected by Western blot analysis. (B) Quantification of Western blot densitometry in panel A. Normalization for loading differences was achieved by dividing the densitometry values for individual bands by the densitometry values for $\beta$-actin in the same lane. (C) Dose-dependent increases of AICD-Gal4-mediated luciferase activity with increasing concentrations of PMA ( 10 hour incubation). Luciferase levels normalized to total cell number using protein concentration. (D) Inhibition of $\alpha$-secretases by TAPI-I ( $20 \mu \mathrm{M}$ for two hours) results in decreases in sAPP $\alpha$, C83-Gal4, and AICDGal4 levels as detected by Western blot analysis. (E) Quantification of Western blot densitometry in panel D and normalized $\beta$-actin levels in the same lane. (F) Dose-dependent decreases in AICD-Gal4-mediated luciferase activity with increasing TAPII concentrations (two hour incubation). For the luciferase experiments, points represent mean normalized luciferase activity (+/- standard error) of three independent trials, with luciferase levels normalized to total cell numbers using SYBR Green. Student's t-tests with sequential Bonferroni correction for multiple comparisons; * indicates $\mathrm{p}<0.05$; ** indicates $\mathrm{p}<0.0 \mathrm{I}$. "Control" uses the same media as the treatments and contains the same amount of DMSO as drug treated cells. 
A

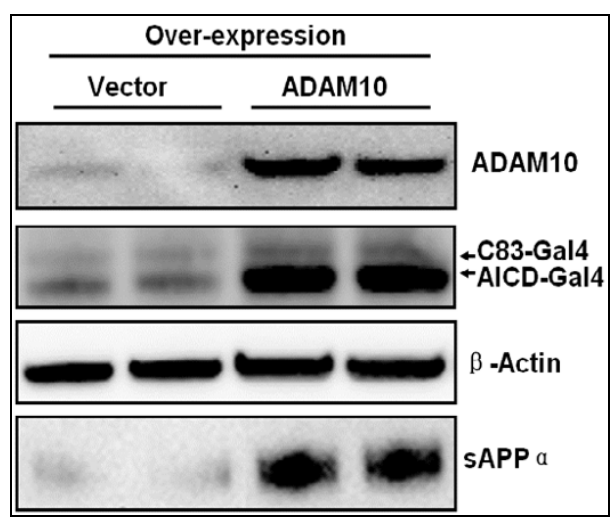

C

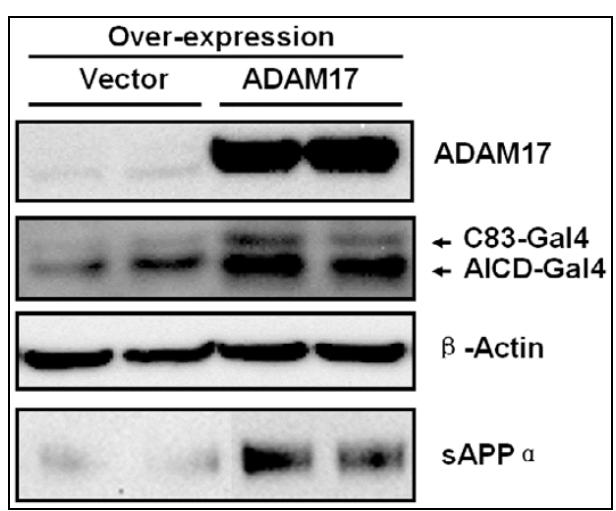

B

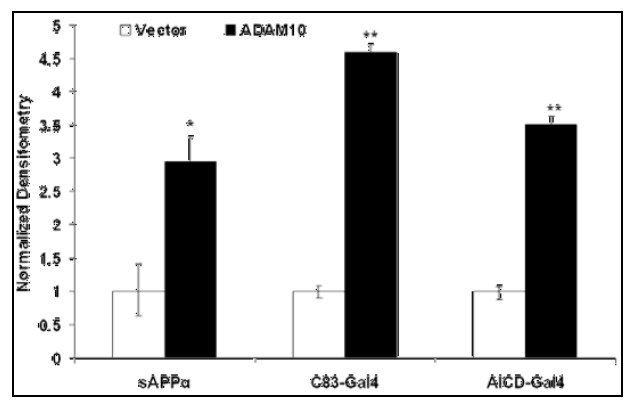

D

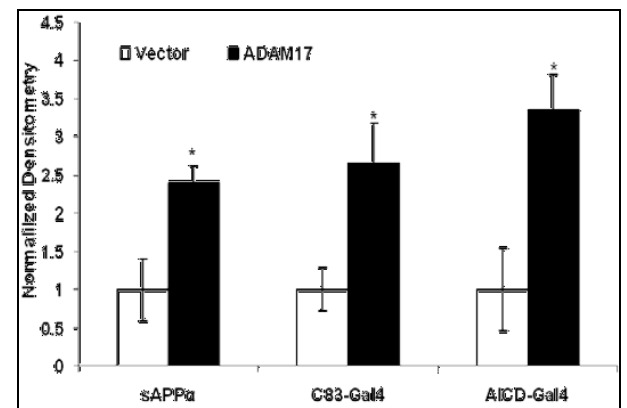

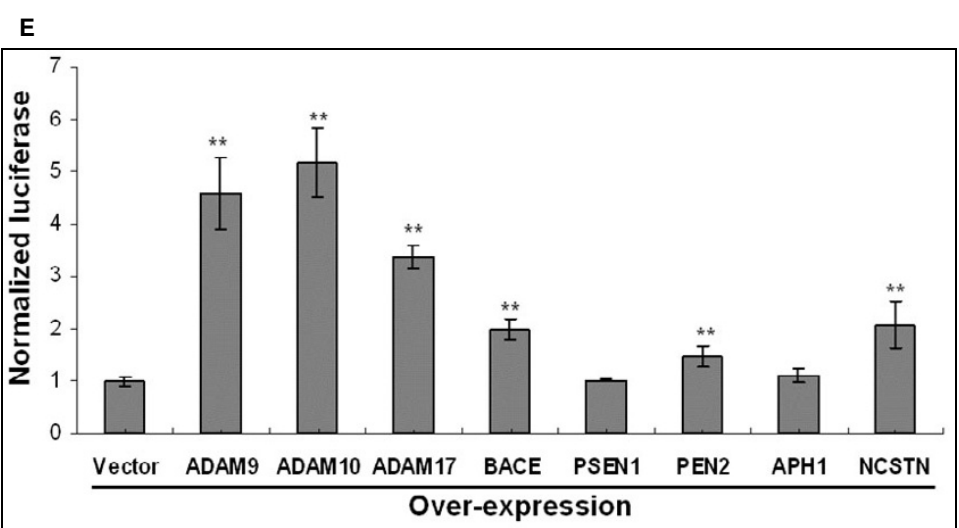

\section{Figure 4}

Over-expression of individual secretase genes in SY5Y-APP-Gal4 cells increases AICD-Gal4 mediated luciferase activity. (A) Transient over-expression of ADAMI0 increases ADAMI0, AICD-Gal4, C83-Gal4, and sAPP $\alpha$ levels compared to cells transfected with empty vector. (B) Quantification of Western blot densitometry in panel A. (C) ADAMI7 transient over-expression significantly increases ADAMI7, AICD-Gal4, C83-Gal4, and sAPP $\alpha$ levels. (D) Quantification of Western blot densitometry in panel C. (E) Transient over-expression of individual secretase genes increases AICD-Gal4 mediated luciferase activity. Luciferase was normalized to transfection efficiency, by dividing by Renilla luciferase activity. Individual secretase over-expression plasmids were co-transfected with PRL-SV40 plasmid, expressing Renilla luciferase. Bars represent the mean normalized luciferase activity of four independent trials and error bars represent standard errors. Statistical significance was determined using two-sample, one-tail t-tests to compare each secretase gene with the empty vector, followed by sequential Bonferroni procedure to adjust for multiple comparisons. * indicates $p<0.05$; ** indicates $p<0.0$ I. 
ditioned media and cell lysates were collected from these cells and utilized for Western blot analyses and luciferase assays. shRNAs specific for APP, ADAM10, and ADAM17 were tested for their ability to knock-down their target genes (Figure 5A, 5B, 5C). Knock-down of these target genes was robust and we have observed significant protein knock-down with at least two different shRNA sequences for each target gene. Consistent with this knock-down of ADAM 10 and ADAM17, sAPP $\alpha$ levels were decreased significantly (Figure 5D \&5E). In addition, Western blot analyses showed AICD-Gal4 levels were also decreased when APP, ADAM10 and ADAM17 were knocked-down (Figure 5E). Knock-down of these target genes also decreased AICD-Gal4 mediated luciferase activity (Figure 5F). Specifically, APP knock-down significantly decreased luciferase activity about $80 \%(\mathrm{p}<0.01)$; furthermore $\alpha$ secretase (ADAM10, and ADAM17) knock-down significantly decreased luciferase activity 40-60\% ( $\mathrm{p}<0.01$ ). Individual $\gamma$-secretase components, PSEN1, Pen2, APH1, and NCSTN, were also knocked-down and this resulted in significant $30-50 \%$ decreases in luciferase activity ( $\mathrm{p}<$ $0.01)$.

\section{Genetic manipulation of Fe65 and Tip60 levels modulates AICD-Gal4 mediated luciferase activity}

To determine if changes in AICD metabolism modulated AICD-Gal4 mediated luciferase activity, we overexpressed and knocked-down Fe65 and Tip60. Transient over-expression of Fe65 significantly increased luciferase activity more than two-fold $(\mathrm{p}<0.01)$, while transient over-expression of Tip60 resulted in a 30\% increase in luciferase activity that was not significant (Figure 6A). Knock-down of Fe65 and Tip60 resulted in a significant $40-50 \%$ decrease in luciferase activity (Figure 6B; $\mathrm{p}<$ $0.01)$.

\section{Ubiquilin I regulates AICD-Gal4 levels}

Having shown that monitoring AICD-Gal4 mediated luciferase activity accurately measures changes in AICD-Gal4 levels induced by changes in secretase activity/levels, we wanted to determine if this approach could detect regulators with a less direct role in APP proteolysis and AICD signaling. We decided to test Ubiquilin 1 because (i) the gene encoding Ubiquilin 1 (UBQLN1) is located in a region of chromosome 9 that displays linkage to $\mathrm{AD}$ in several independent samples [41-46], (ii) a polymorphism in $U B Q L N 1$ modulates AD risk in several independent samples [47-49], (iii) Ubiquilin 1 can modulate APP trafficking to the cell surface in HEK-293 and H4 cell lines [31], and (iv) Ubiquilin 1 can modulate $\gamma$-secretase activity, though the consequences of this modulation on $\gamma$-secretase substrates were not determined [32-34]. Given this, testing Ubiquilin 1 would determine if our genetic screen can detect regulators of APP trafficking and presenilin endoproteolysis. Furthermore, the role of Ubiquilin
1 in APP metabolism regulation has not been previously investigated in SH-SY5Y cells.

SY5Y-APP-Gal4 cells were transfected separately with five different Ubiquilin 1 shRNAs, APP shRNA and the control shRNA. Cell lysates were collected and utilized for luciferase assays. Individually, all five Ubiquilin 1 shRNA constructs significantly decreased luciferase activity. They resulted in 50\% (p<0.01), 60\% ( $\mathrm{p}<0.01), 40 \%(\mathrm{p}<$ $0.01), 60 \%(\mathrm{p}<0.01)$, and $60 \%(\mathrm{p}<0.01)$ decreases in luciferase activity, respectively (Figure 7A) as compared to cells expressing the control shRNA. To confirm the role of Ubiquilin 1 in AICD-mediated transcriptional activity suggested by these results, we transiently over-expressed Ubiquilin 1 in SY5Y-APP-Gal4 cells and measured luciferase activity. We observed that Ubiquilin 1 over-expression resulted in an approximately $90 \%(\mathrm{p}<0.05)$ increase in luciferase activity compared to the empty vector control (Figure 8A).

\section{Ubiquilin I regulates APP and PSI}

To begin to gain insight into the mechanism(s) by which Ubiquilin 1 modulates AICD-Gal4 mediated luciferase expression, we utilized Western blot analysis of cell lysates and conditioned media from SY5Y-APP-Gal4 cells in which Ubiquilin 1 was knocked-down or over-expressed to monitor APP and Ubiquilin 1 metabolism. Specifically, we analyzed cell lysates and conditioned media of cells expressing Ubiquilin 1 shRNA number 2, since transfection with this shRNA led to the largest decrease in luciferase activity. Expression of this shRNA resulted in a robust Ubiquilin 1 knock-down and led to significantly decreased levels of mature full-length APP, immature fulllength APP, AICD-Gal4, C83-Gal4, and sAPP $\alpha$ (Figures 7B \&7C). To determine if Ubiquilin 1-induced changes in APP mRNA levels underlie the observed changes in full length APP levels, we performed real-time, quantitative PCR on SY5Y-APP-Gal4 cells stably expressing either control or Ubiquilin 1 shRNAs. No Ubiquilin 1-induced changes in APP mRNA levels were observed (Figures 7D). This suggests that Ubiquilin 1 regulation of full-length APP levels occurs post-transcriptionally.

We studied Ubiquilin 1 over-expression to determine if we observed the converse effects on APP proteolytic products and full-length APP. Indeed, we observed that Ubiquilin 1 over-expression resulted in increased levels of mature and immature full-length APP, AICD-Gal4, C83Gal4, and sAPP $\alpha$ (Figures 8B \&8C).

The Ubiquilin 1-induced effects on full-length APP are greater on mature APP levels than on immature APP levels (Figure 7C \&8C). This results in a decrease in the ratio of mature to immature full-length APP-Gal4 when Ubiquilin 1 is knocked-down $(\mathrm{p}<0.01$; Figure $7 \mathrm{C})$ and an increase 
A

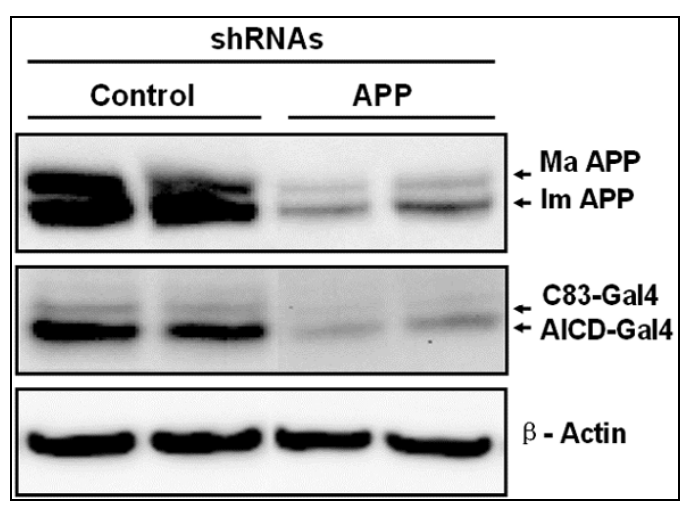

C

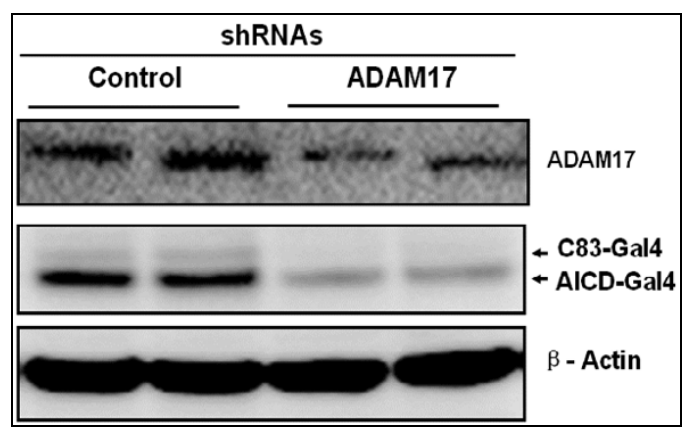

E

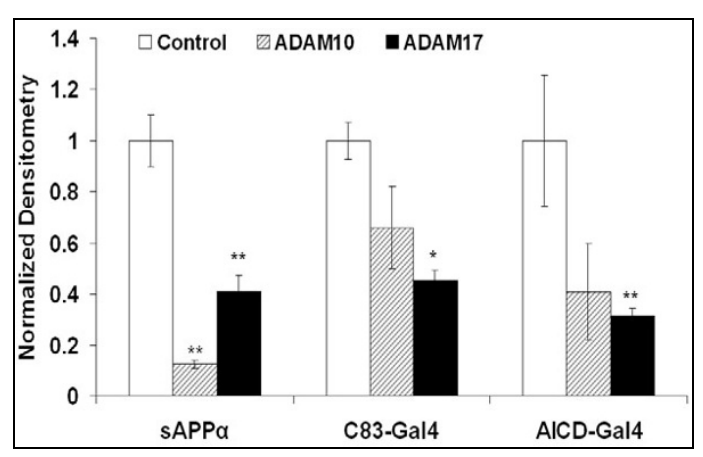

B

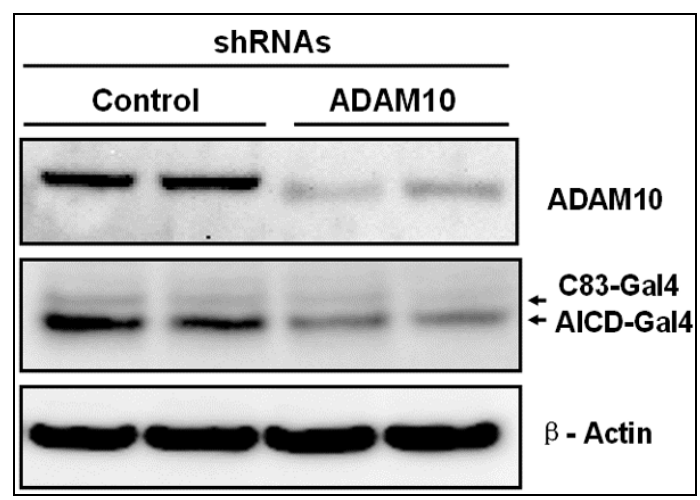

D

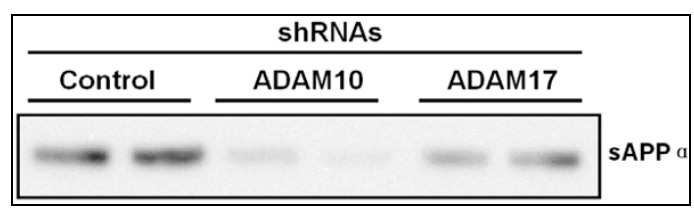

$\mathbf{F}$

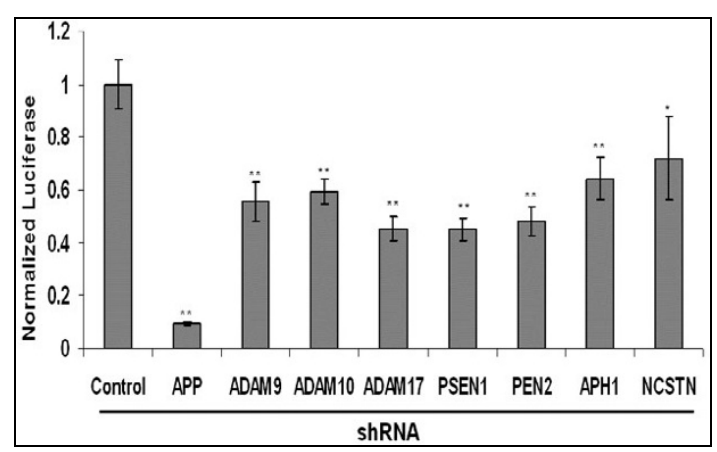

\section{Figure 5}

Knock-down of APP and individual secretase genes in SY5Y-APP-GAL4 cells decreases AICD-Gal4 mediated luciferase activity. (A) APP-specific shRNA decreases full-length APP, C83-Gal4, and AICD-Gal4 levels compared to the control or "non-silencing" shRNA. Results from duplicate transfections with each shRNA are shown. (B) ADAMI0 specific shRNAs decrease endogenous ADAMI0, C83-Gal4, and AICD-Gal4 levels compared to the control shRNA. Results from duplicate transfections with each shRNA are shown. (C) ADAMI7 specific shRNAs decrease endogenous ADAMI7, C83-Gal4, and AICD-Gal4 levels compared to the control shRNA. Results from duplicate transfections with each shRNA are shown. (D) Knock-down of ADAM9, 10, and 17 decrease sAPP $\alpha$ levels compared to control shRNA. (E) Quantification of Western blot densitometries in panels B - D. (F) Transfection with shRNAs specific for APP and individual secretase genes decreases AICDGal4-mediated luciferase expression compared to control shRNA. Bars represent the mean normalized luciferase activity of four independent trials and error bars represent standard errors. Statistical significance was determined using two-sample, one tailed t-tests to compare each secretase shRNA with the control shRNA and sequential Bonferroni procedure to adjust for multiple comparisons. $*$ indicates $p<0.05 ; * *$ indicates $p<0.01$. 
A

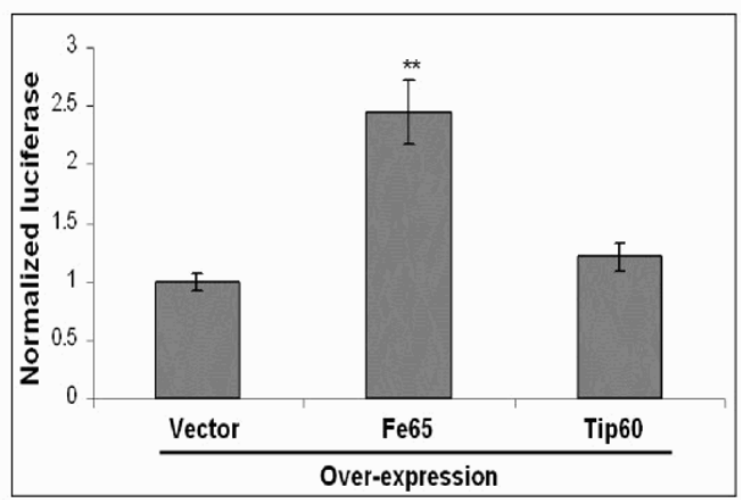

B

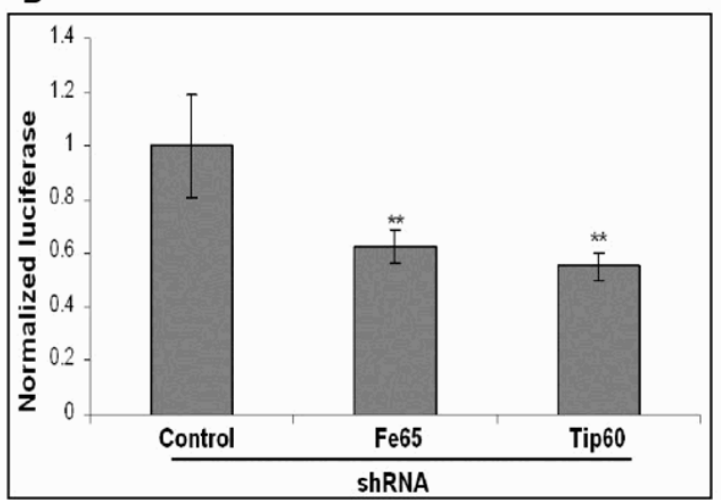

\section{Figure 6}

Genetic alteration of Fe65 or Tip60 levels modulates AICD-Gal4 mediated luciferase activity. (A) Transient overexpression of Tip60 or Fe65 in SY5Y-APP-Gal4 cells increases AICD-Gal4 production compared to empty vector controls. (B) Knock-down of Fe65 or Tip60 in SY5Y-APP-Gal4 cells decreases AICD-Gal4 mediated luciferase activity Bars represent the mean normalized luciferase activity of four independent trials and error bars represent standard errors. Statistical significance was determined using two-sample, one-tailed t-tests to compare each secretase gene and "vector" or "control" and sequential Bonferroni procedure to adjust for multiple comparisons. $*$ indicates $p<0.05$.

in this ratio when Ubiquilin 1 is over-expressed $(\mathrm{p}<0.05$; Figure 8C).

Since Ubiquilin 1 has been reported to regulate PS1 endoproteolysis in HEK-293 cell lines, we sought to determine if the Ubiquilin 1-induced changes that we observed in AICD-Gal4 and C83-Gal4 levels may be due in part to changes in PS1 endoproteolysis [34]. Ubiquilin 1 knockdown in SY5Y-APP-Gal4 cells decreased PS1 carboxy-terminal fragment levels (PS1-CTF; Figure 9A) and Ubiquilin 1 over-expression increased PS1-CTF levels (Figure 9B). We did not observe any changes in the levels of ADAM 10, ADAM 17 or BACE when Ubiquilin 1 was over-expressed or knocked-down (data not shown).

Finally, we over-expressed Ubiquilin 1 in naïve SH-SY5Y to ensure that the results we observed are not limited to the SY5Y-APP-Gal4 cell line. We found that in these naïve cells Ubiquilin 1 over-expression resulted in increased total, mature and immature APP, sAPP as well as PS1 CTF, consistent with our findings in SY5Y-APP-Gal4 cells (data not shown).

\section{Discussion}

Taking advantage of the APP intracellular domain's (AICD) ability to activate transcription, we established an assay to monitor APP metabolism in the human neuroblastoma cell line, SH-SY5Y. We are using this assay in combination with RNAi-mediated knock-down of positional candidate genes as a genetic screen to identify regu- lators of APP metabolism. Here we describe validation of this experimental approach using pharmacologic and genetic modulation of known APP metabolism regulators. We find that AICD-Gal4 mediated luciferase activity is significantly and accurately changed when secretases, Fe65, Tip60, or Ubiquilin 1 levels/activities are modulated pharmacologically or genetically. The ability of Ubiquilin 1 to regulate APP metabolism in SH-SY5Y cells had not been investigated previously. Our initial findings show that in these cells Ubiquilin 1 regulates total APP levels, APP maturation and PS1 endoproteolysis. Our results lead us to conclude that the genetic screen we describe is capable of identifying genes that encode regulators of APP proteolysis, APP maturation, APP levels, and AICD activity.

\section{Validation of AICD-Gal4 luciferase assay}

The functional assay for identifying APP metabolism regulators relies on the ability of an AICD-Gal4 fusion to transactivate a firefly luciferase reporter gene [16]. While the biological role of AICD-mediated transactivation is unclear $[20,23,24,50]$, we utilized this transactivation function purely as a reporter of APP processing and therefore APP metabolism. We determined that monitoring AICD-Gal4 mediated luciferase activity is correlated to AICD-Gal4 levels by utilizing pharmacologic and genetic agents to regulate secretase activities and thereby modulate AICD-Gal4 levels. In SH-SY5Y cells stably expressing an APP-Gal4 fusion protein and a Gal4-UAS driven luciferase reporter construct (SY5Y-APP-Gal4 cells), we uti- 
A

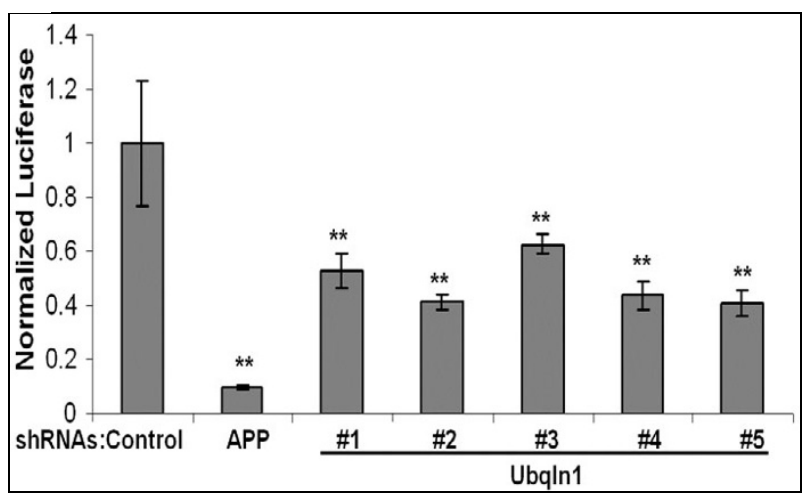

C

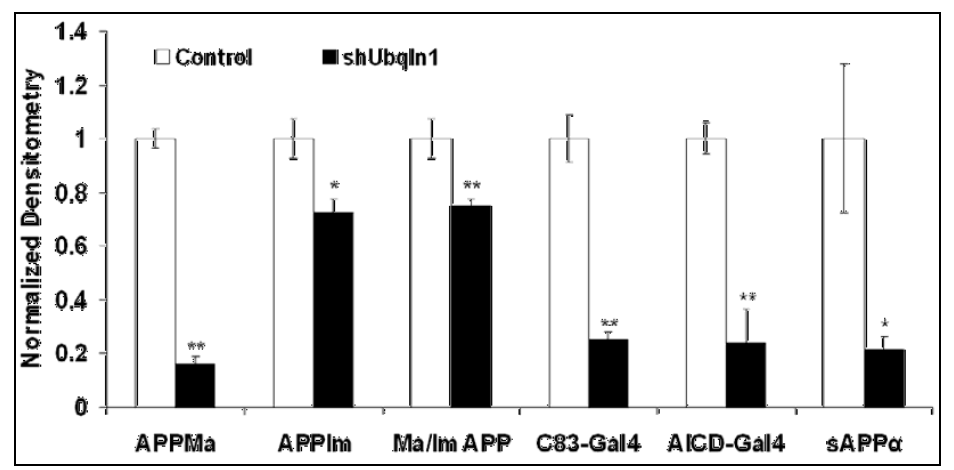

B

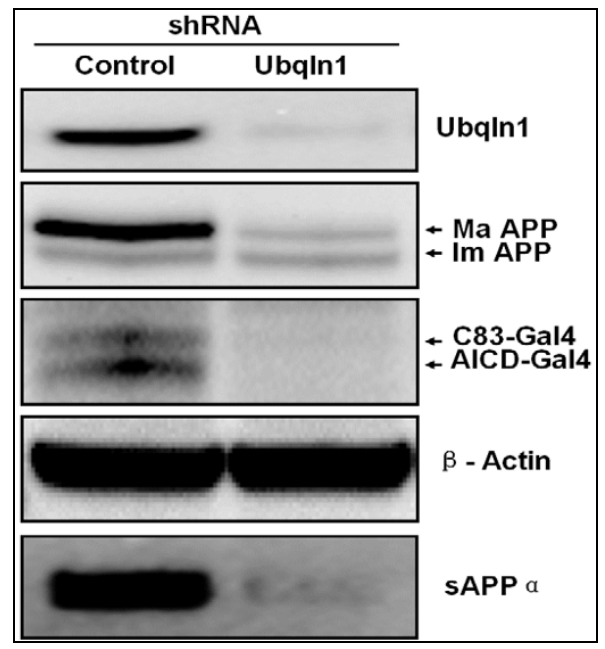

D

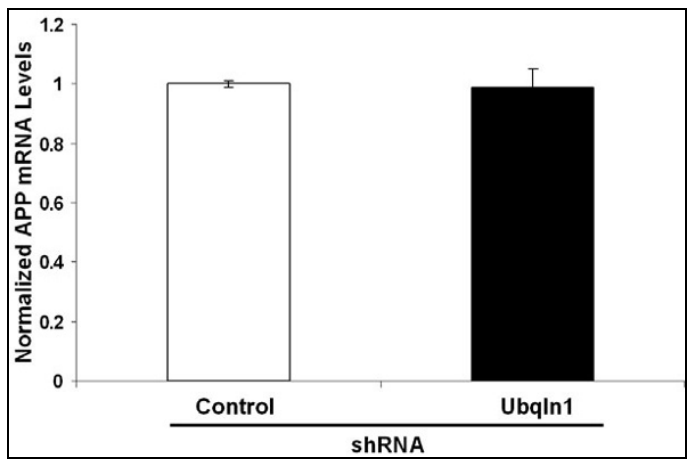

\section{Figure 7}

Ubiquilin I knock-down regulates APP-Gal4 metabolism in SY5Y-APP-Gal4 cells. (A) Ubiquilin I knock-down decreases AICD-Gal4-mediated luciferase activity. SY5Y-APP-Gal4 cells stably expressing the control shRNA, an APP specific shRNA, or five different shRNA targeting Ubiquilin I were generated. Cell lysates were utilized to measure AICD-Gal4 mediated luciferase activity. Bars represent the mean normalized luciferase activity (+/- standard error) of six independent trials. (B) SY5Y-APP-Gal4 cells stably expressing Ubiquilin I specific shRNA (\#2) have decreased Ubiquilin I, mature and immature APPGal4, C83-Gal4, AICD-Gal4, and sAPP $\alpha$ levels compared to cells expressing control shRNA. (C) Quantification of Western blot results. Bars represent mean densitometry (+/- standard error) of three independent trials. Black bars represent the densitometry from Ubiquilin I knock-down cells; white bars represent the densitometry from cells expressing control shRNA. Abbreviations: Ma APP denotes mature APP, Im APP denotes immature APP; Ma/lm APP denotes the mature APP/immature APP ratio. Statistical significance between mock and over-expression for each measure was determined using a two-sample, one tailed t-test and sequential Bonferroni procedure to adjust for multiple comparisons. * indicates $p<0.05$; ** indicates $p<$ 0.0I. (D) Ubiquilin I knock-down does not alter APP mRNA levels compared to control shRNA as measured by quantitative PCR.

lized TAPI-1 and L-685,458 to inhibit $\alpha$ - and $\gamma$-secretases respectively. TAPI- 1 inhibits $\alpha$-secretase cleavage of APP as well as several other cell surface proteins including TNF $\alpha$ [38]. L-685,458 is a potent and selective cell-permeable $\gamma$ secretase inhibitor [51]. Both of these inhibitors decreased AICD-Gal4 levels and decreased AICD-Gal4 mediated luciferase activity to similar levels. Inhibiting BACE activity did not have an appreciable effect on AICD-Gal4 levels or AICD-Gal4 mediated luciferase activity, which is not surprising since the majority of APP processing occurs via the $\alpha$-secretase pathway in SH-SY5Y cells. Stimulation of $\alpha$-secretase using PMA [52] increased AICD-Gal4 levels and increased AICD-Gal4 mediated luciferase activity to similar levels.

To further validate our functional assay, we overexpressed and knocked-down genes that encode the $\alpha-, \beta$ , and $\gamma$-secretases. Similar to the effects of pharmacologic 

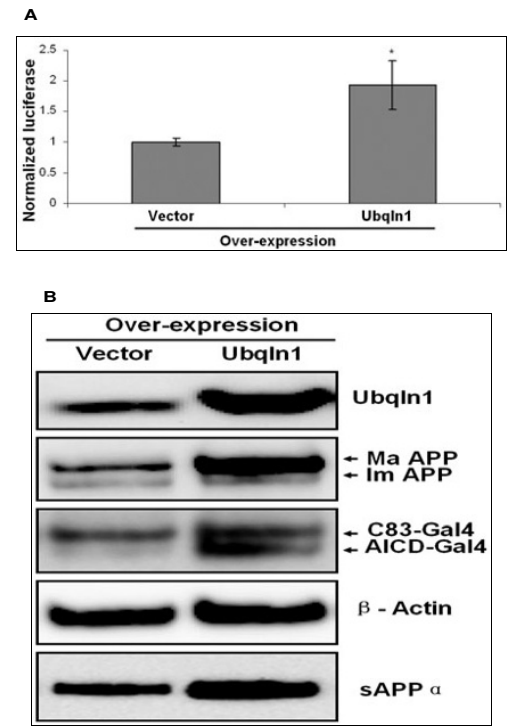

c

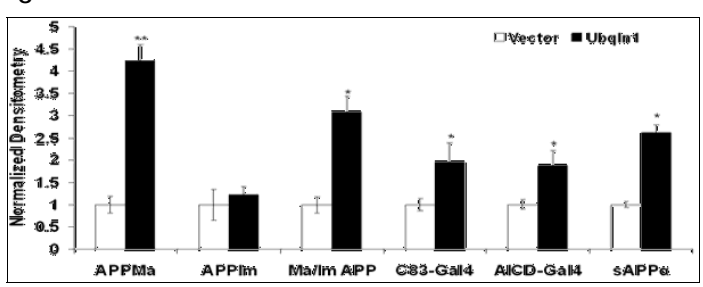

Figure 8

Ubiquilin I over-expression regulates APP-Gal4 metabolism in SY5Y-APP-Gal4 cells. (A) Transient Ubiquilin I over-expression increases AICD-Gal4 mediated luciferase activity. SY5Y-APP-GAL4 cells were transiently cotransfected with UBQLNI over-expression plasmid and a Renilla luciferase over-expression plasmid (pRL-SV40). The latter was used as a transfection efficiency control to normalize AICD-Gal4 mediated luciferase activity. Bars represent the mean normalized luciferase activity (+/- standard error) of six independent trials. Statistical significance was determined using two-sample, one-tailed t-tests to compare each experimental shRNA to the control shRNA and sequential Bonferroni procedure to adjust for multiple comparisons. (B) SY5Y-APP-Gal4 cells transiently over-expressing Ubiquilin I have increased Ubiquilin I, mature and immature APP-Gal4, C83-Gal4, AICD-Gal4, and sAPP $\alpha$ levels compared to vector only cells. (C) Quantification of Western blot results. Bars represent mean densitometry (+/- standard error) of three independent trials. Black bars represent the densitometry from Ubiquilin I over-expressing cells; white bars represent the densitometry from cells expressing empty vector control. Abbreviations: Ma APP denotes mature APP, Im APP denotes immature APP; Ma/lm APP denotes the mature APP/ immature APP ratio. Statistical significance between mock and over-expression for each measure was determined using a two-sample, one tailed t-test and sequential Bonferroni procedure to adjust for multiple comparisons. $*$ indicates $\mathrm{p}<$ 0.05 ; ** indicates $\mathrm{p}<0.01$.
A

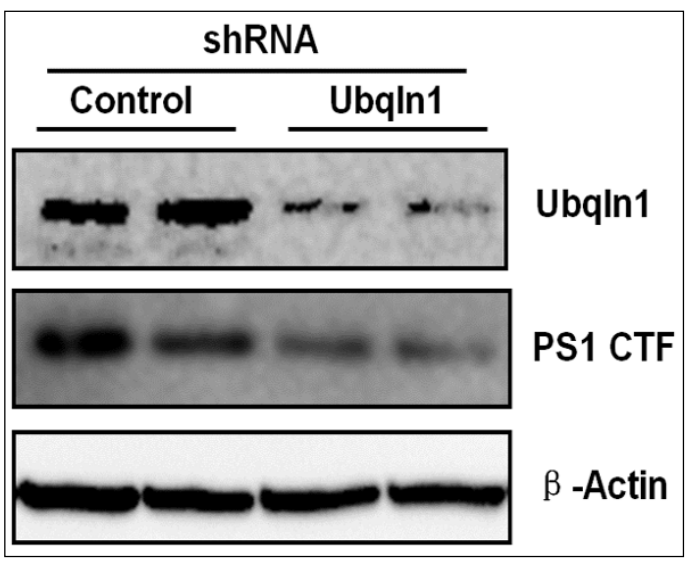

B

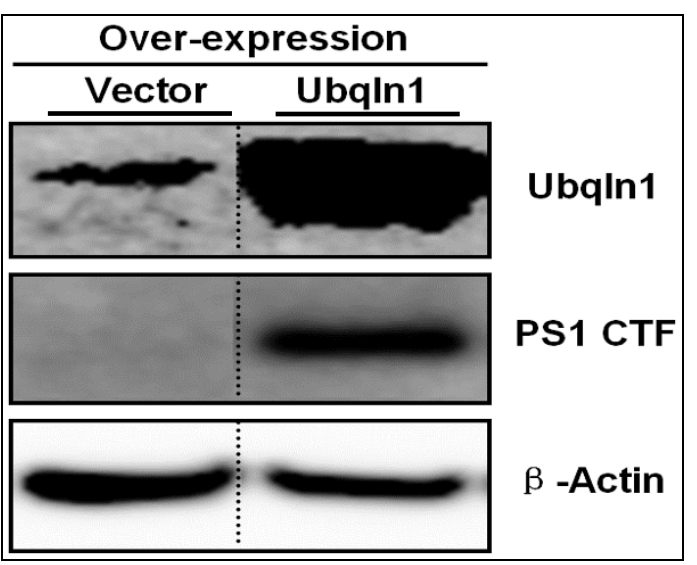

Figure 9

Ubiquilin I regulates Presenilin I endoproteolysis in SY5Y-APP-Gal4 cells.(A) Ubiquilin I knock-down decreases PSI CTF levels in SY5Y-APP-Gal4 cells. (B) Ubiquilin I over-expression increases PSI CTF levels in SY5Y-APPGal4 cells.

modulators of secretases, over-expressing or knockingdown secretase genes resulted in predictable alterations in AICD-Gal4 levels as measured by Western blot analysis. The changes in luciferase activity induced by secretase over-expression or knock-down mirrored the trends observed in the Western blot analysis. Knock-down of APP had the most dramatic effect on AICD-Gal4 mediated luciferase activity while knock-down of genes encoding $\alpha$ and $\gamma$-secretases resulted in significant decreases in AICDGal4 mediated luciferase.

To assess the quality of the AICD-Gal4 mediated luciferase assay we calculated the "Z-factor" for the assay in response 
to the known APP metabolism modulators[53]. The Z-factor is a dimensionless metric that takes assay dynamic range and data variation into consideration to assess the utility and reliability of the assay. Scores between 0.5 and 1.0 indicate an excellent assay [53]. Using the data we collected, we calculated Z-factors for pharmacologic and genetic modulation of the secretases (Table 1 ). For all of these conditions we obtain $Z$ values between 0.5 and 1.0, indicating that our experimental approach is robust and has the capability of identifying APP metabolism regulators that increase or decrease AICD generation.

\section{AICD metabolism regulators modulate AICD-Gal4 luciferase activity}

AICD-Gal4 mediated transactivation has been shown to require Fe65 and Tip60. Fe65 is an adaptor protein that binds to the NPTY sequence in AICD and mediates intracellular trafficking of AICD-Gal4 from the cytoplasm into the nucleus [16]. Once inside the nucleus, the AICD-Gal4/ Fe65 complex recruits the histone acetyltransferase, Tip60. Fe65 and Tip60 are both required for AICD-Gal4 transactivation activity. We observed increased AICDGal4 mediated luciferase activity when we over-expressed Fe65 or Tip60 and decreased luciferase activity when either of these genes was knocked-down.

\section{Ubiquilin I modulates APP metabolism in SH-SY5Y cells} Having validated our experimental approach using direct regulators of APP proteolysis and AICD-metabolism, we then sought to determine if Ubiquilin 1 could modulate AICD-Gal4 mediated luciferase activity. Ubiquilin 1 has been shown to regulate presenilin 1 endoproteolysis and APP trafficking in HEK-293 cells [31-34] and therefore testing Ubiquilin 1 would help to determine whether our experimental approach could detect APP metabolism regulators that are not directly involved in APP proteolysis nor in AICD signaling. When Ubiquilin 1 was knockeddown, AICD-Gal4 luciferase activity was significantly decreased.

Table I: Z-factor values

\begin{tabular}{ccc}
\hline \multicolumn{2}{c}{ APP Metabolism Modulators } & Z factor \\
\hline \multirow{2}{*}{ Pharmacological } & PMA & 0.77 \\
& TAPI-I & 0.63 \\
shRNA & L685,458 & 0.74 \\
& APP & 0.60 \\
\multirow{3}{*}{ Over-expression } & ADAMI0 & 0.71 \\
& ADAMI7 & 0.70 \\
& ADAMI0 & 0.72 \\
& ADAMI7 & 0.60
\end{tabular}

Z-factor values for AICD-Gal4 Luciferase assay calculated when APP metabolism is modulated by pharmacologic or genetic approaches.
Ubiquilin 1 is a conserved protein that contains an $\mathrm{NH}_{2}{ }^{-}$ terminal ubiquitin-like domain (UBL) and a COOH-terminal ubiquitin-associated (UBA) domain [32]. Through these domains, Ubiquilin 1 associates with ubiquitin ligases and the proteosome and is proposed to link ubiquitination with proteosome-mediated protein degradation. This suggests that Ubiquilin 1 plays a role in responding to protein misfolding, aggregation, and/or stress $[32,54]$. In the brains of $\mathrm{AD}$ patients, there is increased Ubiquilin 1 in neurons containing neurofibrillary tangles (NFTs), as compared to control brains [32]. In the brains of Parkinson's disease patients, as well as patients with diffuse Lewy body disease (DLBD), there is strong Ubiquilin 1 staining of Lewy bodies [32]. Finally, a polymorphism in the UBQLN1 gene has been shown to increase AD risk in family-based and large case-control samples [47-49].

The role of Ubiquilin 1 in $\mathrm{AD}$ pathogenesis may be due to its ability to regulate formation of active $\gamma$-secretase complexes and/or regulate APP trafficking [31-34]. Monteiro and colleagues have found that Ubiquilin 1 can regulate full-length Presenilin1 (PS1), Presenilin2 (PS2), Nicastrin, and PEN-2 levels as well as PS1 and PS2 endoproteolysis [32-34]. Specifically, Ubiquilin 1 over-expression increased full-length presenilin (PS1 and PS2) levels in HeLa cells. In HEK-293 cells, Ubiquilin 1 over-expression decreased presenilin endoproteolysis while Ubiquilin 1 knock-down increased presenilin endoproteolysis [34]. In addition, Monteiro and colleagues show that nicastrin and Pen-2 levels are decreased by Ubiquilin 1 over-expression and increased by Ubiquilin 1 knock-down in HEK293. In addition to these effects on $\gamma$-secretase components, Hiltunen and colleagues reported that Ubiquilin 1 knock-down decreased steady-state full-length immature APP levels, increased trafficking of APP from intracellular compartments to the cell surface, and increased steadystate sAPP $\alpha$ levels in HEK-293 and H4 cell lines [31]. These effects on APP levels and secretion altered A $\beta 40$ and A $\beta 42$ levels. However, Ubiquilin 1 knock-down did not alter $\alpha-, \beta-$, or $\gamma$-secretase levels or C83 and C99 levels in these cell lines.

Here we found that in the human neuroblastoma cell line, SH-SY5Y, Ubiquilin 1 regulates total full-length APP, the ratio of mature to immature APP, as well as PS1 endoproteolysis. To arrive at these conclusions, we over-expressed and knocked-down Ubiquilin 1 in SY5Y-APP-Gal4 cells and monitored APP metabolism using Western blot analysis. We found that Ubiquilin 1 knock-down decreased levels of AICD-Gal4, C83-Gal4, sAPP $\alpha$, full-length mature and immature APP, and the ratio of mature to immature APP. Ubiquilin 1 over-expression elicited the opposite effect on the levels of these molecules. 
The fact the ratio of mature to immature APP is altered by Ubiquilin 1 in the absence of APP mRNA level changes suggests that Ubiquilin 1 modulates trafficking through the secretory pathway in SH-SY5Y cells. This conclusion was reach by Hiltunen and colleagues when investigating the role of Ubiquilin 1 on APP metabolism in $\mathrm{H} 4$ and HEK-293 cell lines [31].

Given the existing reports that Ubiquilin 1 regulates PS1 levels and endoproteolysis in HeLa and HEK-293 cells, respectively, we sought to determine if the observed changes in APP processing may be due, in part, to Ubiquilin 1 mediated changes in PS1 metabolism [32-34]. Interestingly, Hiltunen and colleagues did not observe any changes in PS1 levels or endoproteolysis upon transient Ubiquilin 1 knock-down in HEK-293 [31]. In SY5Y-APPGal4 cells, we observed that Ubiquilin 1 knock-down decreases PS1 endoproteolysis and Ubiquilin 1 overexpression promotes PS1 endoproteolysis. Presumably these changes in PS1-CTF levels alter $\gamma$-secretase activity and cleavage of other $\gamma$-secretase substrates. At this time, it is not clear how Ubiquilin 1 regulates PS1 endoproteolysis. No alterations in ADAM10, ADAM17, or BACE levels were observed when Ubiquilin 1 was knocked-down or over-expressed. These results suggest that Ubiquilin 1 regulates APP metabolism not only by controlling the ratio of mature to immature APP but also by post-transcriptionally controlling total APP (mature and immature) levels and PS1 endoproteolysis.

It is interesting to note that the effects of Ubiquilin 1 overexpression/knock-down on APP and presenilin metabolism that we observe in SH-SY5Y cells are different than those observed in HEK-293 and HeLa cells [31,34]. In SHSY5Y cells we find Ubiquilin 1 knock-down decreased total, mature, and immature full-length APP, sAPP $\alpha$, C83 and AICD steady-state levels and the ratio of mature to immature APP, while over-expression increased these same steady-state levels. In addition, Ubiquilin 1 overexpression increased PS1 endoproteolysis. In HEK-293 cells, Hiltunen et al. found that Ubiquilin 1 knock-down decreased steady-state immature full-length APP levels, increased sAPPa levels, and no effects were observed in C83, C99, AICD, and PS1 CTF levels [31]. However, Massey et al. observed an increase in PS1 endoproteolysis in HEK-293 cells [34]. In SH-SY5Y cells however, Ubiquilin 1 seemingly has opposite effects on APP and presenilin metabolism than observed in HEK-293. At this time the reasons for these differences are not clear; they could be due to differences in experimental procedure (e.g. differences in cell confluency, and/or RNAi techniques [transient siRNA versus stable shRNA]) and/or inherent differences in these two cell types. One of the noticeable differences between these cells is that in SH-SY5Y cells, the majority of full-length APP is mature, whereas in HEK-
293 the majority of full-length APP is immature (data not shown). Cell type dependent effects of Ubiquilin 1 have been observed previously. In COS7 cells, Ubiquilin 1 over-expression reduced cell surface expression of nicotinic acetylcholine receptors (nAChRs), while in superior cervical ganglion neurons Ubiquilin 1 over-expression had no effect on nAChR levels [55]. These cell typedependent effects are interesting given the differential vulnerability observed in AD brains, where subsets of neocortical and hippocampal neurons preferentially degenerate [56]. In addition to these cell type-dependent effects, Ubiquilin 1 has been shown to function in seemingly opposite ways. Ubiquilin 1 over-expression has been shown to promote accumulation of some proteins [HASH-1[57], HES-1[57], and $\mathrm{GABA}_{\mathrm{A}}$ receptor[58]] as well as to promote degradation of other proteins [nAChRs[55] and Hepatitis C virus RNA-dependent RNA polymerase [NS5B][59]]. It will be important to study the role of Ubiquilin 1 on APP metabolism in primary neurons and in vivo to determine its true role in regulating APP metabolism and in AD pathogenesis.

Our Ubiquilin 1 results suggest that in SH-SY5Y cells, Ubiquilin 1 regulates APP metabolism not only by controlling the ratio of mature to immature APP but also by post-transcriptionally controlling total APP (mature and immature) levels and PS1-CTF levels.

\section{AICD-Gal4 luciferase assay accurately reports AICD-Gal4 levels}

Finally, we were struck by the ability of the AICD-Gal4 mediated luciferase assay to accurately report AICD-Gal4 levels. To determine if these measures of AICD-Gal4 were correlation, we plotted the change in luciferase activity versus the change in AICD-Gal4 levels as measured by Western blot analysis. AICD-Gal4 levels were modulated by pharmacologic or genetic modulation of secretases and Ubiquilin 1 . This analysis revealed a strong and significant correlation between AICD-Gal4 levels and AICD-Gal4mediated luciferase expression (Figure 10; $\mathrm{R}^{2}=0.95 ; \mathrm{p}=6$ $\left.{ }^{*} 10^{-6}\right)$. The best fit line of this relationship has a slope close of 0.71 demonstrating that this luciferase assay provides an accurate reporter of changes in AICD-Gal4 levels. In addition, measuring AICD-Gal4 mediated luciferase activity provides a simple, quick and inexpensive means for monitoring changes in APP metabolism. The genetic screen we describe can be successfully utilized to identify genes that putatively modulate AICD-Gal4 levels. Additional assays, including as Western blot and ELISA, will be necessary to confirm their role in APP metabolism regulation and gain insight into the mechanism of regulation.

\section{Conclusion}

We have established and validated an AICD-Gal4 based functional assay in SH-SY5Y cells. Using this assay in com- 


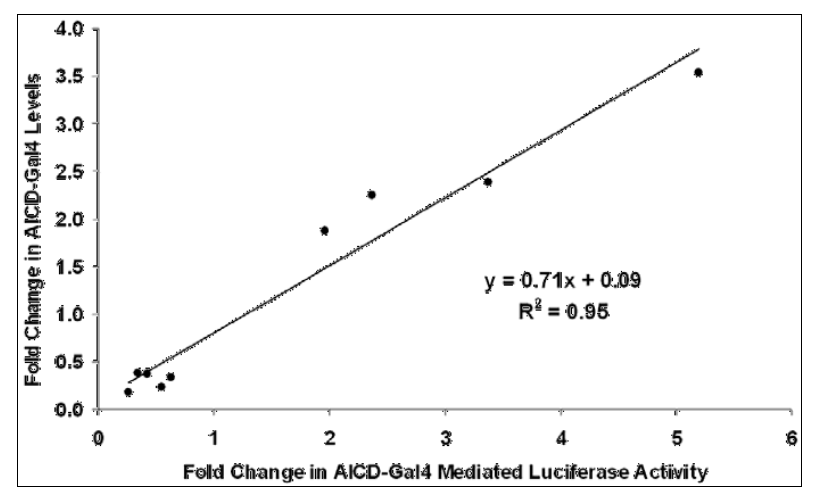

Figure 10

Correlation between AICD-Gal4 mediated luciferase levels and AICD-Gal4 levels determined by Western blot analysis. Using data from pharmacologic (PMA, TAPI- I, L-685,458), over-expression (ADAM I0, ADAMI7, Ubiquilin I) or knock-down (ADAM I0, ADAMI7, Ubiquilin I) mediated modulation of AICD-Gal4 levels we plotted the average fold change in AICD-Gal4 levels versus the average fold change in AICD-Gal4 mediated luciferase activity. For transient over-expression, luciferase activity and AICD-Gal4 levels were normalized to transfection efficiency by Renilla luciferase activity assays. The line is represents the least squares linear regression to this data.

bination with RNAi, we have developed a genetic screen to identify regulators of APP metabolism. This screen accurately, robustly, and easily measures changes in AICD-Gal4 levels. We demonstrate that these AICD-Gal4 levels can be altered by pharmacologic or genetic modulation of genes that directly regulate APP levels, AICD trafficking/signaling, APP maturation, and APP proteolysis. Using this approach, we show that Ubiquilin 1 can regulate AICD-Gal4 levels in SH-SY5Y cells. Ubiquilin 1 regulates AICD-Gal4 levels by modulating APP levels, the ratio of mature to immature APP, and PS1 endoproteolysis. Taken together, our results demonstrate that this genetic screen is capable of identifying APP metabolism regulators that can modulate the APP proteolytic processing, APP maturation, APP levels, and AICD trafficking/signaling.

\section{Methods}

\section{Chemicals and antibodies}

Phorbol 12-myristate 13-acetate (PMA), L-685,458, and puromycin were purchased from Sigma. TAPI-1 was purchased from Peptides International. $\beta$-secretase inhibitor II, N-Benzyloxycarbonyl-Val-Leu-leucinal Z-VLL-CHO, was purchased from Calbiochem. The APP C-terminal antibody (A8717; 1:1000) and $\beta$-actin antibody $(1: 10,000)$ were purchased from Sigma. The 6E10, antiAPP antibody was purchased from Covance and utilized for detection of $\operatorname{sAPP} \alpha(1: 1000)$. The BACE1 antibody
(1:1000) was purchased from Bioscience. The ADAM10 (C-terminal) antibody $(1: 1000)$ was purchased from ProSci. The Ubiquilin 1 antibody (1:160) was purchased from Zymed. The ADAM17 antibody (1:1000) was purchased from Chemicon. The HRP-conjugated secondary antibodies (anti-mouse and anti-rabbit) (1:10,000) were purchased from GE.

\section{Plasmids}

The plasmids APP-Gal4, APP*-Gal4 and Gal4, Gal4-UASluciferase (encoding firefly luciferase) were kindly provided from Dr. Thomas Südof, and are described elsewhere [16]. Briefly, each of these plasmids encodes only the DNA binding domain of Gal4. The ADAM10 overexpression plasmid was kindly provided by Dr. Paul Saftig. The ADAM9 and ADAM17 were provided by Dr. Carl Blobel. The empty vector of ADAM9, ADAM10 and ADAM17 is pcDNA3.1. Ubiquilin 1 over-expression plasmid, which was constructed from pCMV vector, was kindly provided by Dr. Mervyn J. Monteiro.

\section{Cells and cell culture}

SH-SY5Y and naïve human embryonic kidney (HEK)-293 cells were purchased from ATCC. These cell lines were cultured in Dulbecco's modified Eagle's medium (DMEM) supplemented with $10 \%$ fetal bovine serum, $2 \mathrm{mM} \mathrm{L-}$ glutamine, 100 units $/ \mathrm{ml}$ penicillin, and $100 \mu \mathrm{g} / \mathrm{ml}$ streptomycin. SH-SY5Y cells that stably express APP-Gal4, APP*-Gal4, or Gal-4, and carrying the Gal4-UAS-luciferase reporter construct were constructed by co-transfecting one of the Gal4 constructs, the pCDNA3.1 plasmids, along with the Gal4-UAS plasmid, and selecting resistant clones with $400 \mu \mathrm{g} / \mathrm{ml}$ G418. These cells were then tested for $\gamma$-secretase dependent luciferase activity. Clonal lines that stably express luciferase were obtained and were maintained with media containing $200 \mu \mathrm{l} / \mathrm{ml} \mathrm{G418.}$

\section{RNAi}

Plasmid-based shRNA constructs were purchased from Open-Biosystems (Birmingham, AL). These constructs are part of the human retroviral shRNA library housed at the Drexel University RNAi Resource Center. We utilized the following target specific shRNAs: for UBQLN1 shRNAs (Open Biosystems catalog \#s: v2HS_58534, v2HS_254856, v2HS_254715, v2HS_255129 and v2HS_58531); for ADAM9 shRNAs (V2HS_17130, V2HS_17127, V2HS_17126, and V2HS_17129); for ADAM10 shRNAs (v2HS_94294, v2HS_94297, and v2HS_94295); for ADAM17 shRNAs (RHS3979-9619367, RHS3979-9619368, RHS3979-9619369, and RHS39799619370); for BACE1 shRNAs (V2HS_25207, V2HS_25209, V2HS_25206, V2HS_25205, V2HS_25210); for PSEN1 shRNAs (v2HS_89932, v2HS_89931); for PSEN2 shRNAs (v2HS_93093); for APH1 shRNAs(v2HS_117094, v2HS_117096); for NCSTN shR- 
NAs(v2HS_255892). As a negative control shRNA, we utilized the non-silencing shRNA from Open Biosystems, Inc. (RHS 1707). shRNA constructs were transfected using Arrest-In transfection reagent (Open Biosystems, Inc.) using the conditions suggested by the manufacturer. Stably expressing shRNA clones were generated by adding $2 \mu \mathrm{g} /$ $\mathrm{ml}$ puromycin 24 hours post-transfection. Populations of resistant clones were detected five to seven days post-transfection.

\section{Western Blot Analysis}

Cells were lysed in RIPA cell lysis buffer ( $50 \mathrm{mM}$ Tris-HCL pH 7.4, $150 \mathrm{mM} \mathrm{NaCl}, 1 \mathrm{mM}$ EDTA, $1 \%$ NP-40, $1 \mathrm{mM}$ PMSF and $1 \mu \mathrm{g} / \mathrm{ml}$ aprotinin, $1 \mu \mathrm{g} / \mathrm{ml}$ leupeptin, and 1 $\mu \mathrm{g} / \mathrm{ml}$ pepstatin), and centrifuged at 14,000 rpm for 15 minutes at $4{ }^{\circ} \mathrm{C}$. The resulting supernatant was transferred to a new micro-centrifuge tube. The protein concentration of the cell lysates was determined using the BCA protein assay kit (Pierce, Rockford, IL) according to the manufacturer's instructions. Equal quantities of protein were loaded into the wells of $4-12 \%$ Bis-Tris polyacrylamide gels (Invitrogen) along with See Blue plus 2 protein marker (Invitrogen). Gels were run using MES running buffer and transferred to PVDF membrane (Immobilon $\mathrm{PSQ}$, Millipore) using a semi-dry transfer apparatus (Owl Scientific) and NuPage transfer buffer (Invitrogen). PVDF membranes were blocked in TBST with 5\% dry milk for at least two hours, washed extensively, then incubated with primary antibody for either one hour at room temperature or overnight at $4{ }^{\circ} \mathrm{C}$. After removing the primary antibody, membranes were extensively washed and incubated with either goat-anti-rabbit-HRP or goat-anti-mouse-HRP secondary antibodies $(1: 10,000 ; \mathrm{GE})$ for one hour at room temperature. Membranes were washed and developed using West Dura Extended Duration Substrate (Pierce). The blot was visualized using a FluoroChem 8900 imaging system (Alpha Innotech), and signals were quantified using AlphaEase Fc software. To account for any differences in loading, target band densitometries were divided by actin densitometries obtained from the same lane. These corrected densitometries were normalized to controls in each experiment.

Detection of sAPPa followed the protocol detailed in Lanni et al. and Bergamaschi et al. [52,60]. Briefly, conditioned media was collected and $48 \%$ trichloroacetic acid (TCA) was added so that the TCA final concentration was $15 \%$. This mixture was incubated on ice for 30 minutes, and centrifuged at 14,000 rpm for 20 minutes. Following this spin, the supernatant was aspirated and discarded. $500 \mu \mathrm{l}$ of ice cold acetone was used to resuspend the pellet. This mixture was placed at $-20^{\circ} \mathrm{C}$ for at least $30 \mathrm{~min}$ utes, followed by centrifugation at $14,000 \mathrm{rpm}$ for 20 minutes. The supernatant was carefully aspirated and discarded. The remaining pellet was air dried for 10 minutes and then $20 \mu \mathrm{l}$ of RIPA was added and this sample was utilized for Western blot analysis. The sAPP $\alpha$ bands were detected using the 6E10 (1:1000) as the primary antibody.

\section{Luciferase assays}

For firefly luciferase assays, 7,500 cells were plated into the 96 well plates. In an experiment, each treatment was applied to a total of six wells. After treatments, conditioned media was aspirated and discarded. $100 \mu \mathrm{l}$ GLB (Glo Lysis Buffer, Promega) was added to lyse the cells. 30 $\mu \mathrm{l}$ of each cell lysates was transferred to a white plate (Greiner Bio-one), and $30 \mu \mathrm{l}$ Steady-Glo (Promega) was added. Luciferase was measured using a Top-Count Scintillation Counter/Luminescence Reader (Packard, Inc.) Another $30 \mathrm{ul}$ of each cell lysates was transferred to the other white color plate, and $30 \mu \mathrm{l} 20 \times$ SYBR Green (diluted in PBS from Invitrogen 10,000× SYBR Green) was added. SYBR Green fluorescence was measured after 5 minutes incubation in dark using an excitation wavelength of $485 \mathrm{~nm}$, and emission wavelength of $527 \mathrm{~nm}$, and an integration time of 0.1 seconds on a Fluoroscan Ascent FL fluorescence plate reader (Thermo Labsystems, Inc.). The luciferase signal was normalized to cell number by dividing the luciferase signal by the SYBR Green reading for the same well. For dual luciferase assays, SH-SY5YAPP-Gal4 cells that stably express firefly luciferase were co-transfected with pRL-SV40, which constitutively overexpresses Renilla luciferase, along with other plasmids. The dual luciferase assay was performed $24-48$ hours post-transfection. The media was aspirated and discarded. $30 \mu \mathrm{l}$ Dual-Glo luciferase substrate (Promega) was added to lyse the cells. All cell lysates were resuspended and transferred to a white 96 well plate (Greiner Bio-one). After 10 minutes incubation at room temperature, firefly luciferase was measured using a Top-Count Scintillation Counter/Luminescence Reader (Packard, Inc.). Next, $30 \mu \mathrm{l}$ Stop-Glo substrate (Promega) was added to the cell lysates containing Dual-Glo. After 10 minutes room temperature incubation, Renilla luciferase was measured using the same Top-Count Scintillation Counter/Luminescence Reader (Packard, Inc.) For normalization, the firefly luciferase signal was divided by the Renilla luciferase signal for the same well. In an experiment, each treatment was applied to a total of four wells.

\section{RNA Extraction and Real-time, Quantitative PCR}

In triplicate, cells stably expressing control or Ubiquilin 1 specific shRNAs were washed twice with cold PBS and total RNA was isolated using the RNeasy Mini Kit (Qiagen, Inc). cDNA was synthesized using total RNA (3.5 $\mu \mathrm{g})$, N6 random primers $(12.5 \mu \mathrm{M})$ and SuperScript II Reverse Transcriptase (Invitrogen). cDNAs were diluted 1:30 using RNase-free $\mathrm{H}_{2} \mathrm{O}$ to a final concentration of $2 \mathrm{ng}$. Diluted cDNAs were mixed with APP or $18 S$ primer/probe sets (Applied Biosystems, Inc.; APP Catalog \# 
Hs00169098_m1; 18S Catalog \# Hs99999901_s1), 2× PCR Universal Master Mix (Applied Biosystems, Inc.) and amplified using an ABI 7500 Real-Time PCR System following the manufacturer's directions. To determine differences in APP mRNA levels, we utilized the $\Delta \Delta \mathrm{Ct}$ method.

\section{Statistical analysis}

Values in the text and figures are presented as means \pm standard errors of at least three independent experiments. Equal variance or separate variance two-sample student's t-test were used, as appropriate, to compare two groups. Bonferroni correction analysis was used to correct for multiple comparisons within a single experiment.

\section{Competing interests}

RET reports being a consultant or serving on the scientific advisory board and board of directors of Torrey Pines Therapuetics and Prana Biotechnology; holding equity or stock options with Torrey Pines Therapuetics, Prana Biotechnology, and Elan; and having received consulting or lecture fees from Novartis, Aventis Pharma, Eisai, and PureTech Ventures. No other authors reported any potential conflicts of interest. AJS is a shareholder in Torrey Pines Therapuetics.

\section{Authors' contributions}

$\mathrm{CZ}$ and PJK designed and carried out the majority of the experiments, data interpretation, and helped draft the manuscript. RC performed QPCR experiments. TLC created the SH-SY5Y-APP-Gal4 stable cell line, performed initial characterization of the AICD-Gal4 luciferase assay. SS established the AICD-Gal4 assay in 96 well format using Sybr green cell number normalization. SAP helped in the characterization of Ubiquilin 1. CPC performed the initial characterization of the AICD-Gal4 luciferase assay. MOC performed statistical analyses. JCL was involved in the statistical analyses, interpretation of results and preparation of the manuscript. RET was involved in the design of the genetic screen and interpretation of results. AJS designed the genetic screen, coordinated the studies, interpreted the results, and drafted the manuscript. All authors have read and approved the final manuscript.

\section{Acknowledgements}

We would like to thank Dr. Thomas Südhof and colleagues generously sharing the APP-Gal4, APP*-Gal4 and Gal4-UAS plasmids; Dr Paul Saftig for generously sharing the ADAMI0 plasmid; Dr. Carl Blobel for generously sharing ADAM9 and ADAMI 7 plasmids; and Dr. Mervyn Monteiro for generously sharing the Ubiquilin I plasmid. We would also like to thank all the members of the Saunders lab for helpful discussions and technical assistance in the completion of this work. This work was funded by NINDS (AJS), Commonwealth of PA and Drexel University.

\section{References}

I. Ebinu JO, Yankner BA: A RIP tide in neuronal signal transduction. Neuron 2002, 34:499-502.
2. Urban S, Freeman M: Intramembrane proteolysis controls diverse signalling pathways throughout evolution. Curr Opin Genet Dev 2002, I 2:512-8.

3. Brown MS, Ye J, Rawson RB, Goldstein JL: Regulated intramembrane proteolysis: a control mechanism conserved from bacteria to humans. Cell 2000, 100:391-8.

4. Annaert W, De Strooper B: A cell biological perspective on Alzheimer's disease. Annu Rev Cell Dev Biol 2002, I 8:25-5I.

5. Sisodia SS: Biomedicine. A cargo receptor mystery APParently solved? Science 2002, 295:805-7.

6. Hardy J, Selkoe DJ: The amyloid hypothesis of Alzheimer's disease: progress and problems on the road to therapeutics. Science 2002, 297:353-6.

7. Vassar R, Bennett BD, Babu-Khan S, Kahn S, Mendiaz EA, Denis P, Teplow DB, Ross S, Amarante P, Loeloff R, Luo Y, Fisher S, Fuller J, Edenson S, Lile J, Jarosinski MA, Biere AL, Curran E, Burgess T, Louis JC, Collins F, Treanor J, Rogers G, Citron M: Beta-secretase cleavage of Alzheimer's amyloid precursor protein by the transmembrane aspartic protease BACE. Science 1999, 286:735-4I.

8. Kimberly WT, LaVoie MJ, Ostaszewski BL, Ye W, Wolfe MS, Selkoe $D$ J: \{gamma\}-Secretase is a membrane protein complex comprised of presenilin, nicastrin, aph-I, and pen-2. Proc Natl Acad Sci USA 2003.

9. Edbauer D, Winkler E, Regula JT, Pesold B, Steiner H, Haass C: Reconstitution of gamma-secretase activity. Nat Cell Biol 2003, 5:486-8.

10. Thinakaran G, Borchelt DR, Lee MK, Slunt HH, Spitzer L, Kim G, Ratovitsky T, Davenport F, Nordstedt C, Seeger M, Hardy J, Levey AI, Gandy SE, Jenkins NA, Copeland NG, Price DL, Sisodia SS: Endoproteolysis of presenilin $I$ and accumulation of processed derivatives in vivo. Neuron 1996, I7:181-90.

II. Gu Y, Misonou H, Sato T, Dohmae N, Takio K, Ihara Y: Distinct intramembrane cleavage of the beta-amyloid precursor protein family resembling gamma-secretase-like cleavage of Notch. J Biol Chem 200I, 276:35235-8.

12. Yu C, Kim SH, lkeuchi T, Xu H, Gasparini L, Wang R, Sisodia SS: Characterization of a presenilin-mediated amyloid precursor protein carboxyl-terminal fragment gamma. Evidence for distinct mechanisms involved in gamma-secretase processing of the APP and Notch I transmembrane domains. J Biol Chem 200I, 276:43756-60.

13. Sastre M, Steiner H, Fuchs K, Capell A, Multhaup G, Condron MM, Teplow DB, Haass C: Presenilin-dependent gamma-secretase processing of beta-amyloid precursor protein at a site corresponding to the S3 cleavage of Notch. EMBO Rep 200I, 2:835-4I.

14. Cupers P, Orlans I, Craessaerts K, Annaert W, De Strooper B: The amyloid precursor protein (APP)-cytoplasmic fragment generated by gamma-secretase is rapidly degraded but distributes partially in a nuclear fraction of neurones in culture. J Neurochem 200I, 78: I 168-78.

15. Kimberly WT, Zheng JB, Guenette SY, Selkoe DJ: The intracellular domain of the beta-amyloid precursor protein is stabilized by $\mathrm{Fe} 65$ and translocates to the nucleus in a notch-like manner. J Biol Chem 2001, 276:40288-92.

16. Cao X, Sudhof TC: A transcriptionally [correction of transcriptively] active complex of APP with Fe65 and histone acetyltransferase Tip60. Science 2001, 293: I I5-20.

17. Cao X, Sudhof TC: Dissection of amyloid-beta precursor protein-dependent transcriptional transactivation. I Biol Chem 2004, 279:2460I-II.

18. Hass MR, Yankner BA: A \{gamma\}-secretase-independent mechanism of signal transduction by the amyloid precursor protein. J Biol Chem 2005, 280:36895-904.

19. Baek SH, Ohgi KA, Rose DW, Koo EH, Glass CK, Rosenfeld MG: Exchange of N-CoR corepressor and Tip 60 coactivator complexes links gene expression by NF-kappaB and beta-amyloid precursor protein. Cell 2002, I I 0:55-67.

20. Pardossi-Piquard R, Petit A, Kawarai T, Sunyach C, Alves da Costa C, Vincent B, Ring S, D'Adamio L, Shen J, Muller U, St George Hyslop P, Checler F: Presenilin-dependent transcriptional control of the Abeta-degrading enzyme neprilysin by intracellular domains of betaAPP and APLP. Neuron 2005, 46:54I-54.

21. Creaven M, Hans F, Mutskov V, Col E, Caron C, Dimitrov S, Khochbin $\mathrm{S}$ : Control of the histone-acetyltransferase activity of Tip60 
by the HIV-I transactivator protein, Tat. Biochemistry 1999 , 38:8826-30

22. von Rotz RC, Kohli BM, Bosset J, Meier M, Suzuki T, Nitsch RM, Konietzko U: The APP intracellular domain forms nuclear multiprotein complexes and regulates the transcription of its own precursor. J Cell Sci 2004, I $17: 4435-48$.

23. Chen AC, Selkoe DJ: Response to: Pardossi-Piquard et al, "Presenilin-Dependent Transcriptional Control of the Abeta-Degrading Enzyme Neprilysin by Intracellular Domains of betaAPP and APLP." Neuron 46, 54I-554. Neuron 2007, 53:479-83.

24. Herz J: Overview: the long and winding road to understanding Alzheimer's disease. Neuron 2007, 53:477-9.

25. Chen F, Gu Y, Hasegawa H, Ruan X, Arawaka S, Fraser P, Westaway $\mathrm{D}$, Mount $\mathrm{H}$, St George-Hyslop P: Presenilin I mutations activate gamma 42-secretase but reciprocally inhibit epsilon-secretase cleavage of amyloid precursor protein (APP) and S3cleavage of notch. J Biol Chem 2002, 277:3652 I-6.

26. Moehlmann T, Winkler E, Xia X, Edbauer D, Murrell J, Capell A, Kaether C, Zheng H, Ghetti B, Haass C, Steiner H: Presenilin-I mutations of leucine 166 equally affect the generation of the Notch and APP intracellular domains independent of their effect on Abeta 42 production. Proc Natl Acad Sci USA 2002, 99:8025-30

27. Saura CA, Choi SY, Beglopoulos V, Malkani S, Zhang D, Shankaranarayana Rao BS, Chattarji S, Kelleher RJ, Kandel ER 3rd, Duff K, Kirkwood $A$, Shen J: Loss of presenilin function causes impairments of memory and synaptic plasticity followed by age-dependent neurodegeneration. Neuron 2004, 42:23-36.

28. Schroeter EH, llagan MX, Brunkan AL, Hecimovic S, Li YM, Xu M, Lewis HD, Saxena MT, De Strooper B, Coonrod A, Tomita T, Iwatsubo T, Moore CL, Goate A, Wolfe MS, Shearman M, Kopan R: A presenilin dimer at the core of the gamma-secretase enzyme: insights from parallel analysis of Notch I and APP proteolysis. Proc Natl Acad Sci USA 2003, 100: 13075-80.

29. Song W, Nadeau P, Yuan M, Yang X, Shen J, Yankner BA: Proteolytic release and nuclear translocation of Notch-I are induced by presenilin-I and impaired by pathogenic presenilin-I mutations. Proc Natl Acad Sci USA 1999, 96:6959-63.

30. Komano H, Shiraishi H, Kawamura Y, Sai X, Suzuki R, Serneels L, Kawaichi M, Kitamura T, Yanagisawa K: A new functional screening system for identification of regulators for the generation of amyloid beta-protein. J Biol Chem 2002, 277:39627-33.

31. Hiltunen M, Lu A, Thomas AV, Romano DM, Kim M, Jones PB, Xie Z, Kounnas MZ, Wagner SL, Berezovska O, Hyman BT, Tesco G, Bertram L, Tanzi RE: Ubiquilin I modulates amyloid precursor protein trafficking and Abeta secretion. J Biol Chem 2006, 28 I:32240-53.

32. Mah AL, Perry G, Smith MA, Monteiro MJ: Identification of ubiquilin, a novel presenilin interactor that increases presenilin protein accumulation. J Cell Biol 2000, I 5 I:847-62.

33. Massey LK, Mah AL, Ford DL, Miller J, Liang J, Doong H, Monteiro MJ: Overexpression of ubiquilin decreases ubiquitination and degradation of presenilin proteins. J Alzheimers Dis 2004, 6:79-92.

34. Massey LK, Mah AL, Monteiro MJ: Ubiquilin regulates presenilin endoproteolysis and modulates gamma-secretase components, Pen-2 and nicastrin. Biochem J 2005, 391:5 I3-25.

35. Tian G, Ghanekar SV, Aharony D, Shenvi AB, Jacobs RT, Liu X, Greenberg $B D$ : The mechanism of gamma-secretase: multiple inhibitor binding sites for transition state analogs and small molecule inhibitors. J Biol Chem 2003, 278:28968-75.

36. Edbauer D, Willem M, Lammich S, Steiner H, Haass C: Insulindegrading enzyme rapidly removes the beta-amyloid precursor protein intracellular domain (AICD). J Biol Chem 2002, 277:13389-93.

37. Allinson TM, Parkin ET, Condon TP, Schwager SL, Sturrock ED, Turner AJ, Hooper NM: The role of ADAMIO and ADAMI 7 in the ectodomain shedding of angiotensin converting enzyme and the amyloid precursor protein. Eur J Biochem 2004, 271:2539-47.

38. Slack BE, Ma LK, Seah CC: Constitutive shedding of the amyloid precursor protein ectodomain is up-regulated by tumour necrosis factor-alpha converting enzyme. Biochem J 200I, 357:787-94.
39. Abbenante G, Kovacs DM, Leung DL, Craik DJ, Tanzi RE, Fairlie DP: Inhibitors of beta-amyloid formation based on the betasecretase cleavage site. Biochem Biophys Res Commun 2000, 268: I33-5.

40. Paddison PJ, Silva JM, Conklin DS, Schlabach M, Li M, Aruleba S, Balija V, O'Shaughnessy A, Gnoj L, Scobie K, Chang K, Westbrook T, Cleary M, Sachidanandam R, McCombie WR, Elledge SJ, Hannon G]: A resource for large-scale RNA-interference-based screens in mammals. Nature 2004, 428:427-3I.

4I. Blacker D, Bertram L, Saunders AJ, Moscarillo TJ, Albert M, Wiener H, Perry R, Collins JS, Harrell LE, Go RCP, Mahoney A, Beaty T, Fallin MD, Avramopoulos D, Chase GA, Folstein MF, Mclnnis MG, Bassett SS, Doheny KJ, Pugh EW, Tanzi RE: Results of a High Resolution Genome Screen of 437 Alzheimer's Disease Families. Human Molecular Genetics 2003, 1 2:23-32.

42. Holmans $P$, Hamshere $M$, Hollingworth $P$, Rice $F$, Tunstall N, Jones $S$, Moore P, Wavrant DeVrieze F, Myers A, Crook R, Compton D, Marshall H, Meyer D, Shears S, Booth J, Ramic D, Williams N, Norton N, Abraham R, Kehoe P, Williams H, Rudrasingham V, O'Donovan M, Jones L, Hardy J, Goate A, Lovestone S, Owen M, Williams J: Genome screen for loci influencing age at onset and rate of decline in late onset Alzheimer's disease. Am J Med Genet B Neuropsychiatr Genet 2005, 135:24-32.

43. Lee JH, Cheng R, Santana V, Williamson J, Lantigua R, Medrano M, Arriaga A, Stern Y, Tycko B, Rogaeva E, Wakutani $Y$, Kawarai T, St George-Hyslop P, Mayeux R: Expanded genomewide scan implicates a novel locus at $3 q 28$ among Caribbean hispanics with familial Alzheimer disease. Arch Neurol 2006, 63:159|-8.

44. Myers A, Wavrant De-Vrieze F, Holmans P, Hamshere M, Crook R, Compton D, Marshall H, Meyer D, Shears S, Booth J, Ramic D, Knowles H, Morris JC, Williams N, Norton N, Abraham R, Kehoe P, Williams $H$, Rudrasingham $V$, Rice $F$, Giles $P$, Tunstall $N$, Jones $L$, Lovestone S, Williams J, Owen MJ, Hardy J, Goate A: Full genome screen for Alzheimer disease: Stage II analysis. Am J Med Genet 2002, I | 4:235-44.

45. Olson JM, Goddard KA, Dudek DM: A second locus for very-lateonset Alzheimer disease: a genome scan reveals linkage to $20 p$ and epistasis between 20p and the amyloid precursor protein region. Am J Hum Genet 2002, 71 : I54-61.

46. Pericak-Vance MA, Grubber J, Bailey LR, Hedges D, West S, Santoro L, Kemmerer B, Hall JL, Saunders AM, Roses AD, Small GW, Scott WK, Conneally PM, Vance JM, Haines JL: Identification of novel genes in late-onset Alzheimer's disease. Exp Gerontol 2000, 35:1343-52

47. Bertram L, Hiltunen M, Parkinson M, Ingelsson M, Lange C, Ramasamy K, Mullin K, Menon R, Sampson AJ, Hsiao MY, Elliott KJ, Velicelebi G, Moscarillo T, Hyman BT, Wagner SL, Becker KD, Blacker D, Tanzi RE: Family-based association between Alzheimer's disease and variants in UBQLNI. N EnglJ Med 2005, 352:884-94.

48. Kamboh MI, Minster RL, Feingold E, DeKosky ST: Genetic association of ubiquilin with Alzheimer's disease and related quantitative measures. Mol Psychiatry 2006, I I:273-9.

49. Slifer MA, Martin ER, Haines JL, Pericak-Vance MA: The ubiquilin I gene and Alzheimer's disease. N Engl J Med 2005, 352:2752-3. author reply $2752-3$

50. Hebert SS, Serneels L, Tolia A, Craessaerts K, Derks C, Filippov MA Muller U, De Strooper B: Regulated intramembrane proteolysis of amyloid precursor protein and regulation of expression of putative target genes. EMBO Rep 2006, 7:739-45.

51. Shearman MS, Beher D, Clarke EE, Lewis HD, Harrison T, Hunt P, Nadin A, Smith AL, Stevenson G, Castro JL: L-685,458, an aspartyl protease transition state mimic, is a potent inhibitor of amyloid beta-protein precursor gamma-secretase activity. Biochemistry 2000, 39:8698-704.

52. Lanni C, Mazzucchelli M, Porrello E, Govoni S, Racchi M: Differential involvement of protein kinase $C$ alpha and epsilon in the regulated secretion of soluble amyloid precursor protein. Eur J Biochem 2004, 27 I:3068-75.

53. Zhang JH, Chung TD, Oldenburg KR: A Simple Statistical Parameter for Use in Evaluation and Validation of High Throughput Screening Assays. J Biomol Screen 1999, 4:67-73.

54. Heir R, Ablasou C, Dumontier E, Elliott M, Fagotto-Kaufmann C, Bedford FK: The UBL domain of PLIC-I regulates aggresome formation. EMBO Rep 2006, 7:1252-8. 
55. Ficklin $M B$, Zhao $S$, Feng G: Ubiquilin-I regulates nicotineinduced up-regulation of neuronal nicotinic acetylcholine receptors. J Biol Chem 2005, 280:34088-95.

56. Morrison JH, Hof PR: Selective vulnerability of corticocortical and hippocampal circuits in aging and Alzheimer's disease. Prog Brain Res 2002, 136:467-86.

57. Persson $\mathrm{P}$, Stockhausen MT, Pahlman S, Axelson $\mathrm{H}$ : Ubiquilin-I is a novel HASH-I-complexing protein that regulates levels of neuronal bHLH transcription factors in human neuroblastoma cells. Int J Oncol 2004, 25: I2 I3-2I.

58. Bedford FK, Kittler JT, Muller E, Thomas P, Uren JM, Merlo D, Wisden W, Triller A, Smart TG, Moss S): GABA(A) receptor cell surface number and subunit stability are regulated by the ubiquitin-like protein Plic-I. Nat Neurosci 200I, 4:908-16.

59. Gao L, Tu H, Shi ST, Lee KJ, Asanaka M, Hwang SB, Lai MM: Interaction with a ubiquitin-like protein enhances the ubiquitination and degradation of hepatitis C virus RNA-dependent RNA polymerase. J Virol 2003, 77:4|49-59.

60. Bergamaschi S, Binetti G, Govoni S, Wetsel WC, Battaini F, Trabucchi M, Bianchetti A, Racchi M: Defective phorbol ester-stimulated secretion of beta-amyloid precursor protein from Alzheimer's disease fibroblasts. Neurosci Lett 1995, 20 I:I-5.

Publish with Bio Med Central and every scientist can read your work free of charge

"BioMed Central will be the most significant development for disseminating the results of biomedical research in our lifetime. "

Sir Paul Nurse, Cancer Research UK

Your research papers will be:

- available free of charge to the entire biomedical community

- peer reviewed and published immediately upon acceptance

- cited in PubMed and archived on PubMed Central

- yours - you keep the copyright

Submit your manuscript here:

http://www.biomedcentral.com/info/publishing_adv.asp
BioMedcentral 